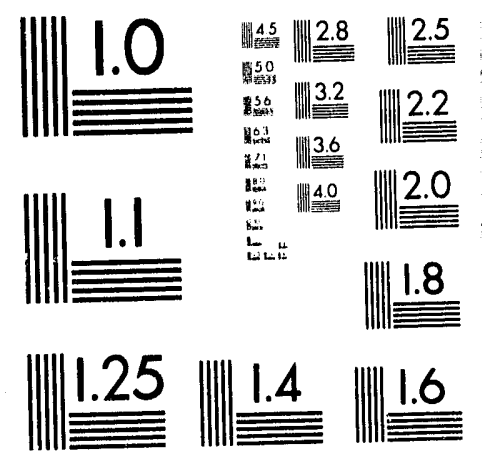



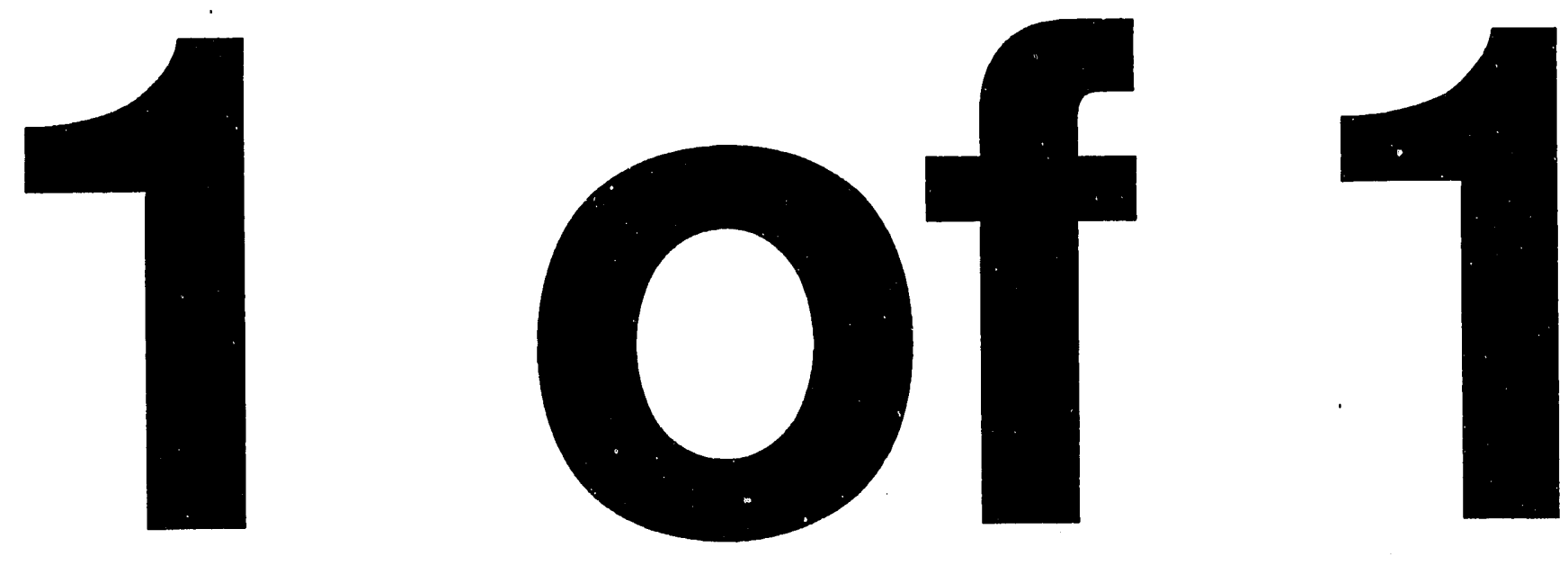


\title{
PREDICTION OF THERMODYNAMIC PROPERTIES OF COAL DERIVATIVES
}

Final Technical Report for Grant Period

March 1, 1991 to February 28, 1994

Marc D. Donohue

Department of Chemical Engineering The Johns Hopkins University

Baltimore, Maryland 21218

September 1993

\author{
Prepared for
}

CHEMICAL SCIENCES DIVISION OFFICE OF BASIC ENERGY SCIENCES THE U.S. DEPARTMENT OF ENERGY AGREEMENT NO. DE-FG02-87ER13777-A005 


\section{N O T I C E}

This report was prepared as an account of work sponsored by the United States Government. Neither the United States nor the Department of Energy, nor any of their employees, nor any of their contractors, subcontractors, or their employees, makes any warranty, expressed or implied, or assumes any legal liability or responsibility for the accuracy, completeness, or usefulness of any information, apparatus, product or process disclosed or represents that its use would not infringe privately-owned rights.

\section{DISCLAIMER}

\footnotetext{
This report was prepared as an account of work sponsored by an agency of the United States Government. Neither the United States Government nor any agency thereof, nor any of their employees, makes any warranty, express or implied, or assumes any legal liability or responsibility for the accuracy, completeness, or usefulness of any information, apparatus, product, or process disclosed, or represents that its use would not infringe privately owned rights. Reference herein to any specific commercial product, process, or service by trade name, trademark manufacturer, or otherwise does not necessarily constitute or imply its endorsement, recomme United States Government or any agency thereof. The views mendation, or favoring by the United States Government or any agency thereof.
} 


\begin{abstract}
The purpose of this research program is to understand and model the effects of different intermolecular forces on the thermodynamic properties of systems containing pure compounds and mixtures. The compounds under consideration vary considerably in size, shape and energy. Therefore in order to develop a theory capable of describing accurately the thermodynamic properties and phase behavior of such systems over a wide range of temperature and pressure, one has to take into account explicitly the differences in shape and size among the various compounds as well as the different type of intermolecular interactions.
\end{abstract}

Over the course of this project, we have developed new equations of state for purecomponent chain molecules. We have shown that the excellent performance of complicated theories, such as the Generalized Flory Dimer (GFD) theory can be mimiced by simpler equations, if certain assumptions for the shape parameters are made. We developed engineering correlations based on the GFD theory, using local composition theory to take into account attractive forces. During the current contract period, we compared various methods for the calculation of the repulsive and attractive contributions to the equation of state against computer simulation data for hard and square-well chains, and against experimental data from the literature. We also have studied microstructure and local order in fluids that contain asymmetric molecules. We developed a theormodynamic model for polar compounds based on a site-site interaction approach. We have shown the equivalence of various classes of theories for hydrogen bonding, and used this equivalence to derive a multiple site model for water. In addition, simple cubic equations of state have been applied to calculate physical and chemical-reaction equilibria in nonideal systems.

In order to obtain a better understanding of the intermolecular forces and to test some of our recent models, we have performed considerable experimental work. We measured infinite dilution activity coefficients using HPLC. We also measured high pressure vapor liquid equilibria of ternary and quaternary systems containing supercritical solvents. In addition, we have used FT-IR spectroscopy to examine the self-association of aliphatic alcohols due to hydrogen bonding, and to investigate the hydrogen bonding in polymer-solvent mixtures.

In the future, we plan to extend our theoretical models for pure components to a more general study of local compositions and non-random effects in mixtures of molecules that differ in molecular size, shape, and type of interaction. 


\section{TABLE OF CONTENTS}

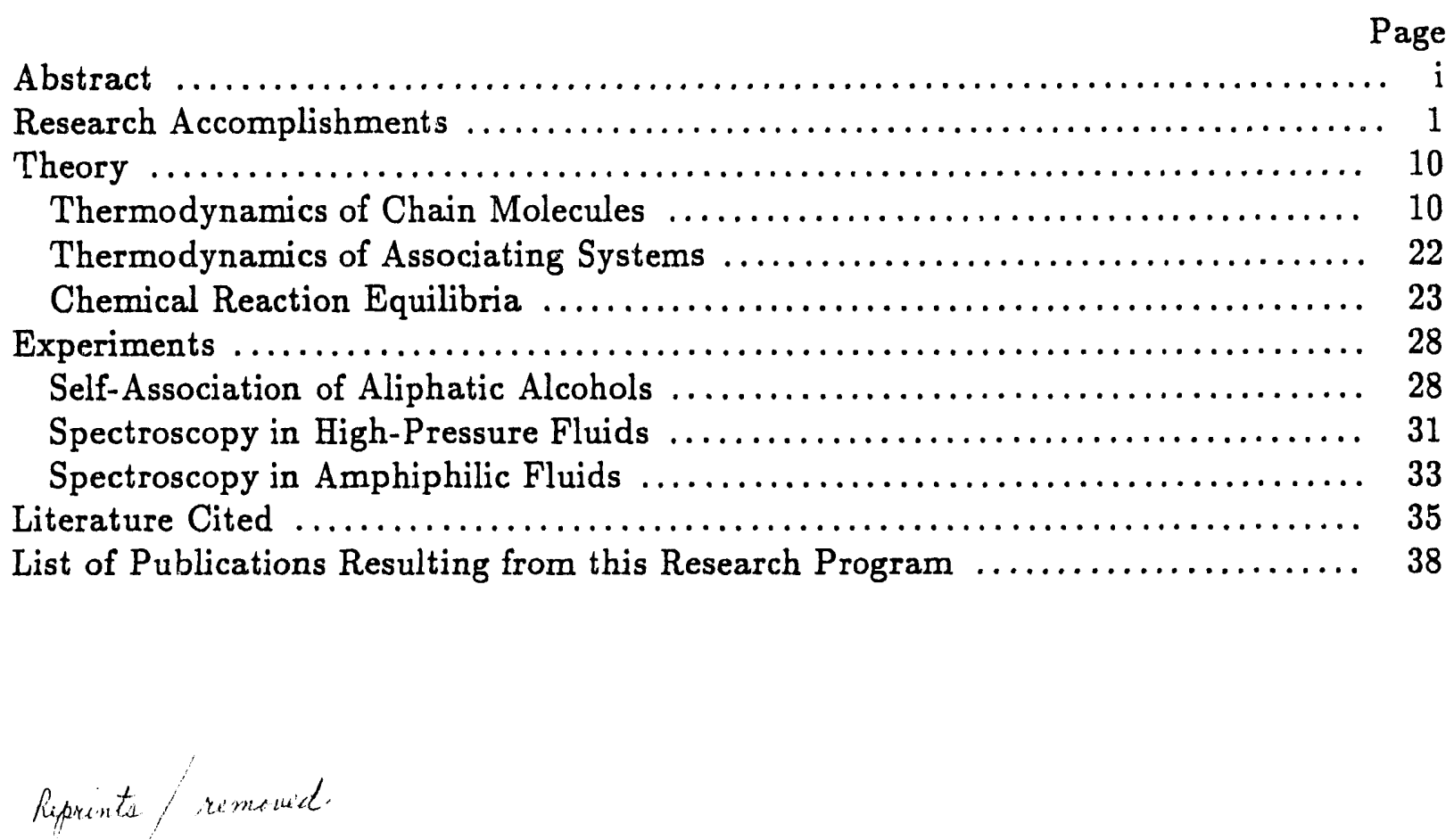




\section{Research Accomplishments}

In this section we present briefly our research accomplishments over the period of this grant. Details are presented in the papers referenced. Copies of these papers are enclosed.

- We have developed a site-site interaction theory to describe the thermodynamics of solutions containing polar and non-polar compounds. In this site-site interaction approach, molecules are considered to be made of sites that interact with each other and with sites on other molecules. A site is either an atom or a group of atoms that can be characterized by its potential function. These sites are identified by their physical properties (size, polarizability, dipole or quadrupole moment, and acid-base properties) and their positions with respect to other sites on the same molecule. One advantage in using this approach is that the sites come within site dimensions of each other and that the physical characteristics of a site are not smeared over the whole molecule. In contrast, in a molecular interaction approach the molecules come within molecular dimensions of each other and the molecule has net properties which are contributions of all the sites that make up the molecule. Another advantage of the site-site interaction approach is that it is applicable to multicomponent mixtures without using any additional mixing rules or parameters, and therefore a wide variety of compounds and mixtures can be treated. Details of the derivation and application of this theory are given in "Thermodynamics of Short Chain Polar Compounds" by Walsh et al., (1991).

- We have made a rigorous comparison of the thermodynamic models for hydrogen bonding systems. Three different classes of theories have been developed specifically to treat hydrogen-bonding systems. Theories in the first class are based on the assumption that hydrogen bonding results in the formation of new species and are referred to as "chemical theories". Theories in the second class are based on lattice-fluid theory that is used to describe different types of specific interactions, and are considered "quasi-chemical" 
generalized for multicomponent associating systems resulting in closed-form expressions that eliminate the infinite series presented in earlier work (Economou et al., 1990). Details about these expressions are given by "Closed-Form Expressions for "Chemical Theory" of Associating Mixtures" by Cämpbell et al. (1992).

- In many equations of state for associating fluids the assumption is made that physical and chemical interactions are separable. We have shown that chemical and physical interactions are coupled and one can not assume a priori that each can be calculated separately and their arbitrary separation leads to a thermodynamic inconsistency. This is discussed in "Thermodynamic Inconsistencies in and Accuracy of Chemical Equations of State for Associating Fluids" by Economou and Donohue (1992a).

- We also have shown that in order to develop an equation of state for associating fluids one must use an equation of state for the "physical" interactions between the species present in the fluid and assume combining rules in order to calculate the parameters of the associating species relative to the parameters of the monomer. With this equation, the fugacity coefficients of the species involved in the chemical equilibria can be evaluated. The expressions for the fugacity coefficients can be substituted in the expression for the chemical equilibria in order to solve the chemical and physical equilibria simultaneously. Details are presented in "Comments on "Thermodynamic Inconsistencies in and Accuracy of Chemical Equations of State for Associating Fluids"' by Anderko et al., (1993).

- We extended the Associated-Perturbed-Anisotropic-Chain-Theory (APACT) to com" pounds with three associating sites per molecule. Details of the derivation and results are discussed later and in "Equation of State with Multiple Associating Sites for Water and Water - Hydrocarbon Mixtures" by Economou and Donohue (1992b).

- We determined the local composition effects on the properties of the pure fluid. Using the square-well potential, we derived an expression for the local composition that was incorporated into the Simplified-PHCT (SPHCT). The new equation is slightly more com- 
the correct second virial coefficient limit, as the density approaches zero. For this purpose, we used computer simulation data for the second virial coefficient of hard chains by Hall and co-workers. The resulting expression is very accurate over the entire density range: from the ideal gas limit to liquid-like densities. It also is applicable to both small molecules and polymers. Details of the derivation of this theory are presented in "Thermodynamic Properties of Hard-Chain Molecules" by Bokis et al. (1993a).

- The GFD theory has been extended by Yethiraj and Hall (1991a) to chain molecules that interact through site-site square-well intermolecular potential. They showed that both the repulsive and the attractive term of the equation of state have the same dependence on the size of the molecule. Thus, they developed a theory for square-well chains by incorporating the Ornstein-Zernike equation for: monomers and the Reference Interaction Site Model (RISM) for dimers. Their equation, though in excellent agreement with simulation data, is very complicated and not explicit with respect to the pressure (it requires a numerical solution at each point of interest). We have developed simple expressions for the attractive term of square-well monomers and dimers based on local composition theory, which, when incorporated into the GFD theory, result in a simple, closed-form equation of state. This theory is in very good agreement with simulation data, and also suitable for engineering calculations. The derivation of this theory is discussed in "Local Composition Model for Square-Well Chains Using the Generalized Flory Dimer Theory" by Bokis et al. (1992).

- We have calculated the site-site pair correlation functions of chain molecules using molecular dynamics simulations. The results can be used to evaluate the parameters of the equation of state by Walsh et al., (1991). Details are presented in A Parame.terization of the Configurational Energy for Short-Chain Molecules by Thomas et al., (1993a).

- In addition to chain molecules that consist of tangent hard spheres, we also have studied chains of various bond lengths. For this purpose, we have performed Monte Carlo simu- 
lations for square-well dimers for bond lengths that are similar to those in real molecules (chlorine and ethane). We developed analytic expressions for the equation of state for square-well dimers using both local composition theory and perturbation theory. Comparison of these theories with the simulation data showed very good agreement. Simulation results and details of the derivation of the theories are presented in "Square-well dimers of different bond lengths: Theory and simulation for the equation of state" by Thomas and Donohue (1993a).

- We also have studied the effect of the bond length in square-well chain molecules longer than dimers. Our goal was to develop an accurate equation of state for homonuclear chains, using molecularly based equations for the repulsive and attractive contributions. Four methods to calculate the compressibility factor for the reference hard-chain term were compared. In order to take into account the effect of chain length on the attractive term, two methods were considered: the GFD theory, and the ei-model, a model based on a site-site approach assuming that chain molecules are made of two kinds of segments: end and internal. To test these theories, we performed simulations for chain molecules with various bond lengths over a wide range of temperatures and densities. The $e i$-model is in very good agreement with the simulation data for both freely-jointed, tangent square-well chain molecules, and fused square-well chain molecules. Furthermore, this model showed very good agreement with experimental data for $n$-alkanes with carbon numbers ranging from 4 to 20. Details of this work are discussed in "An equation of state for chain molecules using a site-interaction model: Theory, simulation and correlation of experimental data" by Thomas and Donohue (1993b).

- We have developed a framework for calculating the thermodynamic properties of each site on a chain molecule. The difference between this approach and other theories, such as PHCT (Donohue and Prausnitz, 1978), GFD (Honnell and Hall, 1989), and SAFT (Chapman et al., 1990), is that in our model, the bond energy is formally assigned to the bonding between segments and belongs to the molecule as a whole. This distinction 
between segmental and bond energies is unimportant in analysis of molecular properties; however, proper assignment of the bond energy to molecular segments is necessary for a coherent definition of the segmental chemical potential and access to submolecular information. This formalism provides a method for predicting the formation of microscopically-ordered regions within a macroscopic bulk phase. The simplest of asymmetrical molecules (a dimer consisting of a hard-sphere site and a square-well site) was used to illustrate fluid microstructure with this sphere-and-bond model. Details of this model are discussed in "Local Order and Microphase Formation in Fluids Containing Asymmetric Molecules" by Kuespert and Donohue (1993a).

- Using this sphere-and-bond formalism, we have developed a thermodynamic model of local ordering in amphiphilic chain fluids, using the Flory equation for the entropy of mixing and the mean-field approximation for the attractive energy. We have applied this model to systems of an amphiphilic chain dissolved in a monomeric solvent and we studied the bulk and microphase equilibria of this system. Details about this model and results are presented in "Local Ordering in Asymmetric Chain Fluids" by Kuespert and Donohue (1993b).

- We have developed a method that combines chemical-reaction equilibrium and phase equilibrium in multicomponent, non-ideal systems. This method is applicable in any fluid or condensed phase and over a wide range of temperatures and pressures. Commonly used cubic equations of state were used to calculate the chemical and phase equilibria for the water-gas shift reaction, the thermal decomposition of methylcyclohexane, and the reaction of carbon monoxide and hydrogen sulfide. Details of this method are discussed later and in "Equation-of-State Calculations of Chemical Reaction Equilibrium in Non-Ideal Systems" by Economou et al. (1993).

- We have shown the importance of accurate second virial coefficients in phase equilibrium calculations, especially calculations of dew points. We developed a model for the second virial coefficient of chain molecules, similar to that from the square-well potential. This 
new expression compares very well with simulation data and with experimental data for $n$-alkanes. Details about this model and analytic results are discussed later and in "Second Virial Coefficients for Chain Molecules" by Bokis et al. (1993b).

- We have compared various equations of state for chain molecules with computer simulation data and with pure-component experimental data. Directly using simulation data, we have developed a new equation of state for chain molecules, which is in very good agreement with experimental data for real systems. Details about this new, simplified GFD equation of state and results from pure-component data reduction are discussed later and in "Application of a Modified Generalized Flory Dimer Theory to Normal Alkanes" by Bokis et al., (1993c).

- We have measured the retention times of solutes in solvent mixtures using High Performance Liquid Chromatography (HPLC). From this data, the ratio of infinite dilution activity coefficients, for a particular solute in two different solvents can be determined. Using this in conjunction with data from Gas Liquid Chromatography, activity coefficients can be calculated. The results obtained compare well with the activity coefficients reported in the litarature. The results also were compared with predictions from different models including UNIFAC, NRTL and APACT. More details of the experimental procedure and results are given in "Measurement of Infinite Dilution Activity Coefficients Using High Performance Liquid Chromatography" by Economou et al., (1991b).

- We investigated hydrogen bonding between solvent molecules and between solvent and polymer molecules using FTIR spectroscopy. The solvents used in this work were methanol, chloroform and tetrahydrofuran. Methanol can self-associate and solvate whereas chloroform and tetrahydrofuran can only solvate. The polymer used was a polyketone capable of solvating with molecules having acidic properties. In addition, hydrogen bonding between an ether with five bonding sites per molecule and methanol was examined. The spectroscopic results were modeled using chemical theory expressed 
in terms of segment-segment interactions. Details of this work are presented in "Hydrogen Bonding in Polymer-Solvent Mixtures" by Economou et al. (1991c).

- We measured high-pressure vapor-liquid equilibria of several ternary and quaternary systems containing anisole, benzaldehyde, methanol, supercritical carbon dioxide and supercritical ethane. The phase equilibria of the systems containing carbon dioxide are measured at $373 \mathrm{~K}$ and pressures up to $22 \mathrm{MPa}$ whereas for the systems containing ethane measurements were made at the same temperature and pressure up to $12 M P a$. Anisole and benzaldehyde hydrogen bond with methanol and therefore for the systems where methanol was present, the solubility of these aromatic hydrocarbons increased. Quantitative studies of the hydrogen bonding between the aromatic hydrocarbons and methanol were made using FTIR spectroscopy. Details of the experimental work and results are presented in "High Pressure Vapor-Liquid Equilibria of Aromatic Hydrocarbons with Carbon Dioxide and Ethane" by Reilly et al. (1992a).

- A novel spectral analysis technique has been developed for the determination of peak areas from spectra in which there are multiple overlapping peaks. The technique involves a combination of Fourier spectral analysis and profile modeling. It has been applied to the problem of determining, from FT-IR spectra, the peak areas that correspond to monomeric and hydrogen-bonded species. The Voigt profile has been found to be better than either the Lorentzian or Gaussian profiles for FT-IR spectra. Details of this spectral analysis technique are discussed in "Analysis of FT-IR spectroscopic data: the Voigt profile" by Reilly et al. (1992b).

- FT-IR spectroscopy has been applied for the determination of the self-association of aliphatic alcohols. Analysis of FT-IR spectroscopic data enables one to determine the species present in a mixture, as well as the equilibrium constants. A qualitative analysis of aliphatic alcohol spectra revealed that there are at least three types of hydrogen bonds occuring in the mixture. In addition, the formation of these bonds did not occur until a threshold value of the alcohol concentration was reached. These results 
can be used to test various theories for hydrogen bonding. More details and results of the spectral analysis for alcohols of various chain lengths and at different temperatures are presented in "Analysis of the self-association of aliphatic alcohols using Fourier transform infrared (FT-IR) spectroscopy" by Thomas et al. (1993). This manuscript will be submitted for publication before the end of the current contract period.

\section{THEORY}

In this section we discuss in detail some of the important aspects of our theoretical research over the course of this grant period. In particular, we discuss our work on chain molecules, on hydrogen bonding systems, and on chemical reaction equilibrium.

\section{Therniodynamics of Chain Molecules}

Equations of state that provide accurate representation of the properties of small, spherically symmetric molecules are not accurate in describing the properties of chain molecules, including polymers. This is because chain molecules have rotational and vibrational motions, some of with depend on density and hence, affect the equation of state and other configurational properties.

Recently, a large amount of research has been focused on deriving theoretical models for chain molecules. Figure 1 shows a comparison of several such theories with simulation data for freely jointed, tangent hard 8-mer and 16-mer molecules. Four theories are included in the comparison: the Perturbed-Hard-Chain theory (PHCT; Beret and Prausnitz, 1975; Donohue and Prausnitz, 1978), the Generalized Flory theory (GF; Dickman and Hall, 1986), the Generalized Flory Dimer theory (GFD; Honnell and Hall, 1989), and the Statistical Associating Fluid theory (SAFT; Chapman et al., 1990). PHCT was derived from phenomenological arguments, and the parameter ' $c$ ' in the repulsive term 
has to be fitted to the simulation data. The GF theory has the same form as PHCT; the difference is that the size parameter $c$ (or $\alpha$ in GF) is calculated from molecular geometry and does not need to be correlated to the data. Both GFD and SAFT take into account the effect of the formation of hard chains. In Figure 1, one sees that the GF equation overpredicts the compressibility factors of hard 8-mers and 16-mers over the entire density range. The PHCT model, although the parameter $c$ was fitted to the simulation data, is not very accurate. It overpredicts the data at low densities, and it underpredicts it at high densities. However, GFD and SAFT give a very good representation of the compressibility factors over the whole density range.

Figure 2 shows the compressibility factors for square-well 4-mers, calculated with the GF, GFD, SAFT, and PHCT models at two values of the reduced temperature. Comparison is made with the Monte Carlo simulation data of Yethiraj and Hall (1991b). The GF theory overpredicts the data over the entire density range, as was the case for hard chains. SAFT, although quite accurate for hard chains, as Figure 1 illustrated, is not very accurate for the square-well molecules. This is because it does not take into account the effect of the formation of chains in the attractive term. The GFD theory, which explicitly takes into account the fact that chain molecules consist of connected segments, has the best performance. PHCT also appears to be in good agreement with the simulation data. Since calculations for the repulsive term with PHCT were not in good agreement with simulations (Figure 1), this suggests that a cancellation of errors occurs between the repulsive and attractive terms in PHCT.

The comparison of calculations using these four theories to simulation data shows that the GFD theory, which rigorously takes into account the formation of the chains in both the repulsive and attractive terms, can predict the properties of chain molecules with reasonable accuracy.

Since the GFD theory is able to accurately describe both the repulsive and the attractive part of the equation of state separately, it can be used as a tool in developing 


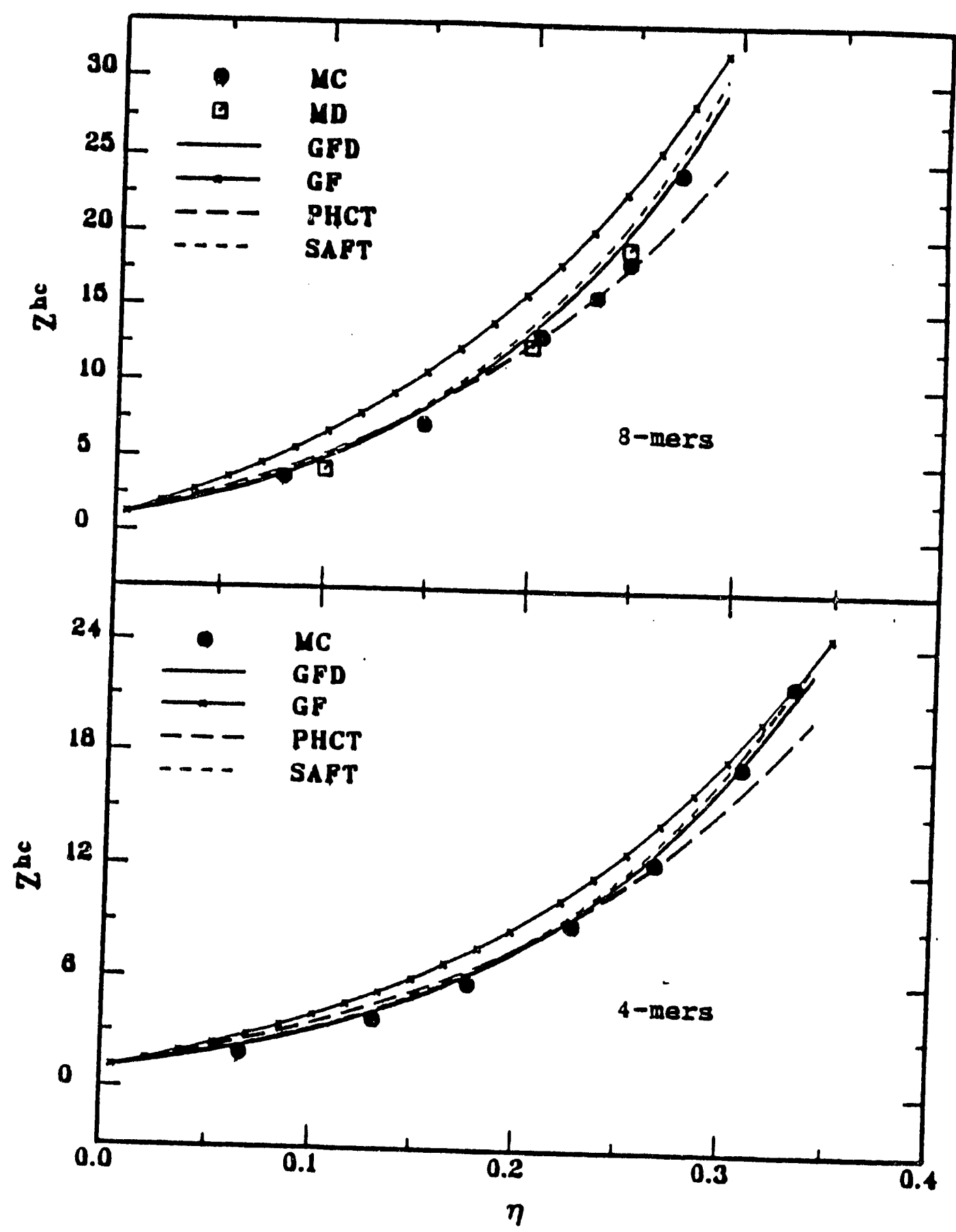

Figure 1. Comparison of various theories for hard-chain molecules. Monte Cario sir ulations of Dickonan and Hall (1988) and molecular dynamics simulations of Dealing. and Hall (1990) also are shown. 


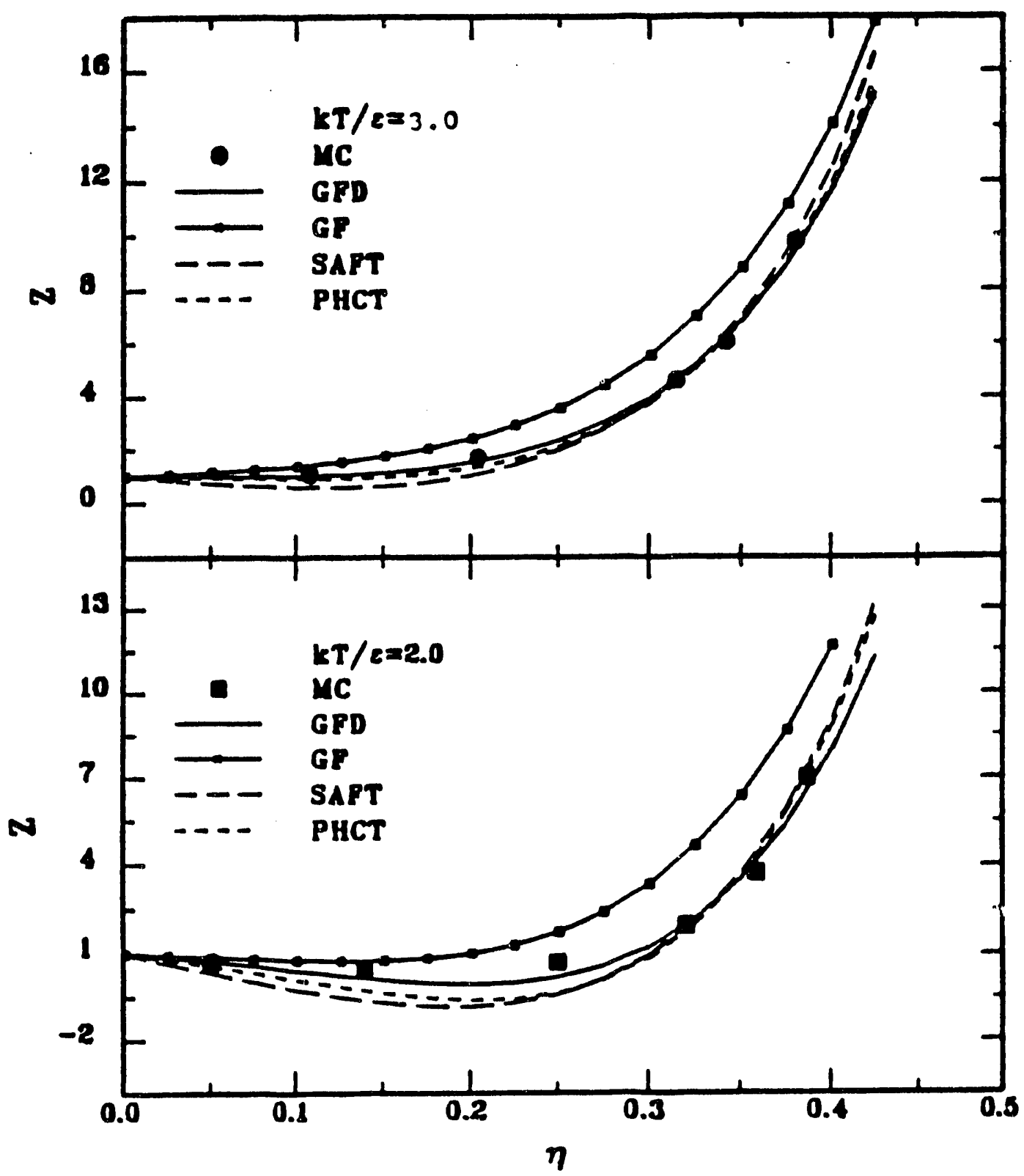

Figure 2. Comparison of various theories for square-well molecules. Monte Carlo simulation data of Yethiraj and Hall (1991) for square-well 4-mers also are includeç. 
a new, simplified expression. Figure 3 shows the ratio of the compressibility factor for chains to that for spherical molecules. The chain compressibility factors were calculated by the GFD equation. One sees that this ratio varies linearly with the reduced density for the repulsive term, and approximately linearly for the attractive term. In addition, the slopes and intercepts vary linearly with chain length; therefore, a simplified version of the GFD theory can be obtained, which has a similar form as PHCT. The difference between this simplified GFD and PHCT is that in PHCT, the size parameters $c$ and $q$ are constant for each species, whereas in the simplified GFD, they depend linearly on density.

Figure 4 shows the same ratio for the repulsive term of the equaticn, calculated from the GFD, SAFT, GF, and PHCT models. Monte Carlo simulation data of Dickman and Hall (1988) also are included. One sees that the simulation data show a similar trend with GFD calculations; however, the slopes and intercepts are different from those predicted by GFD. The same behavior is observed for the attractive contribution, as shown in Figure 5. In this graph, Monte Carlo simulation data for square-well chains are plotted together with calculations from the four theories. We have improved the simplified version of the GFD theory, by fitting the size parameters $c$ and $q$ to linear functions of density and chain length, using the simulation data directly.

To test the applicability of this new, simplified GFD equation, as compared with the other theories, we fit pure-component experimental data. We found that this new model gives the rnost accurate fits. PHCT, which assumes density independent size parameters in both the repulsive and attractive contributions, is the least accurate. Our equation of state is of the same order of accuracy with GFD; however, it is simpler than GFD and therefore, more appealing for engineering calculations. Figure 6 shows typical results from vapor pressure calculations. The Peng-Robinson equation of state also is included for comparison. One sees that errors obtained with our simplified GFD equation of state are close to experimental uncertainty. 


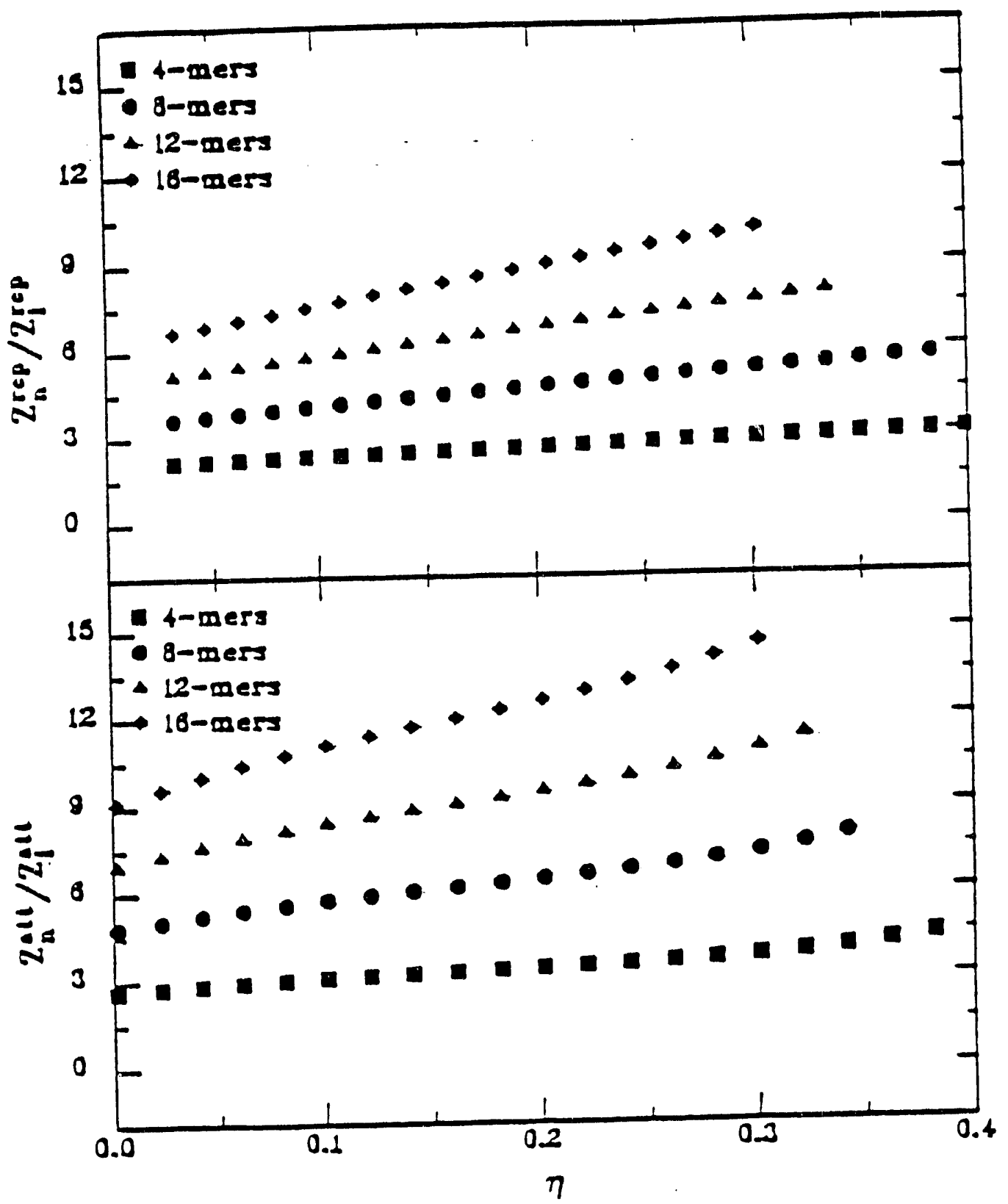

Figure 3. The ratio of compressibility factor for chains to that for spherical molecules, calculated by the GFD equation. A linear variation with respect to density is observed for both the repulsive and attractive contributions. The slopes and intereepts also vary lineariy with chain length and with each other. 


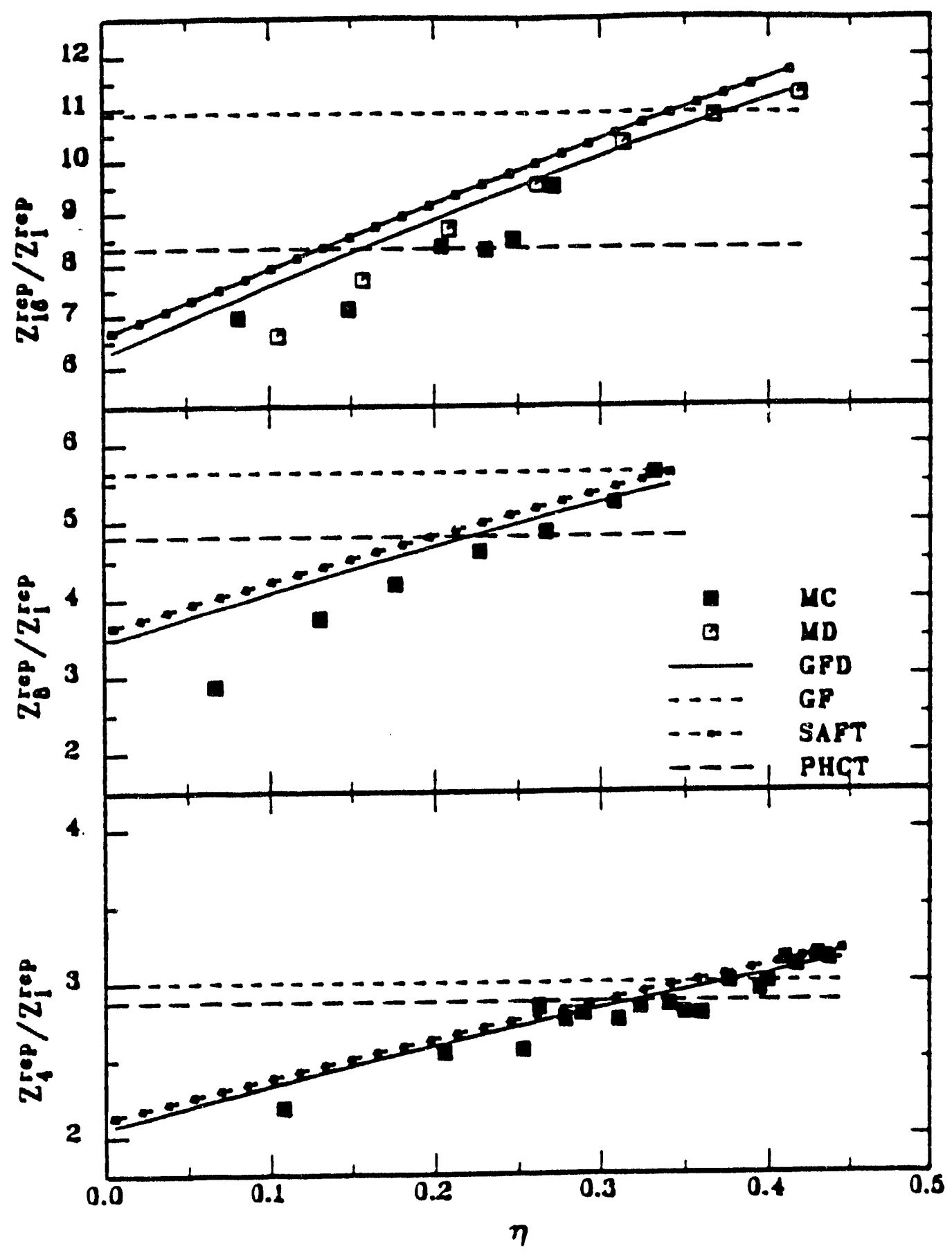

Figure 4. Ratio of repulsive contribution to the compressibility factor for chains to that for monomers, calculated by the GF, GFD, SAFT, and PHCT models. Simulation data of Dicieman and Hall (1988) also are presented. 


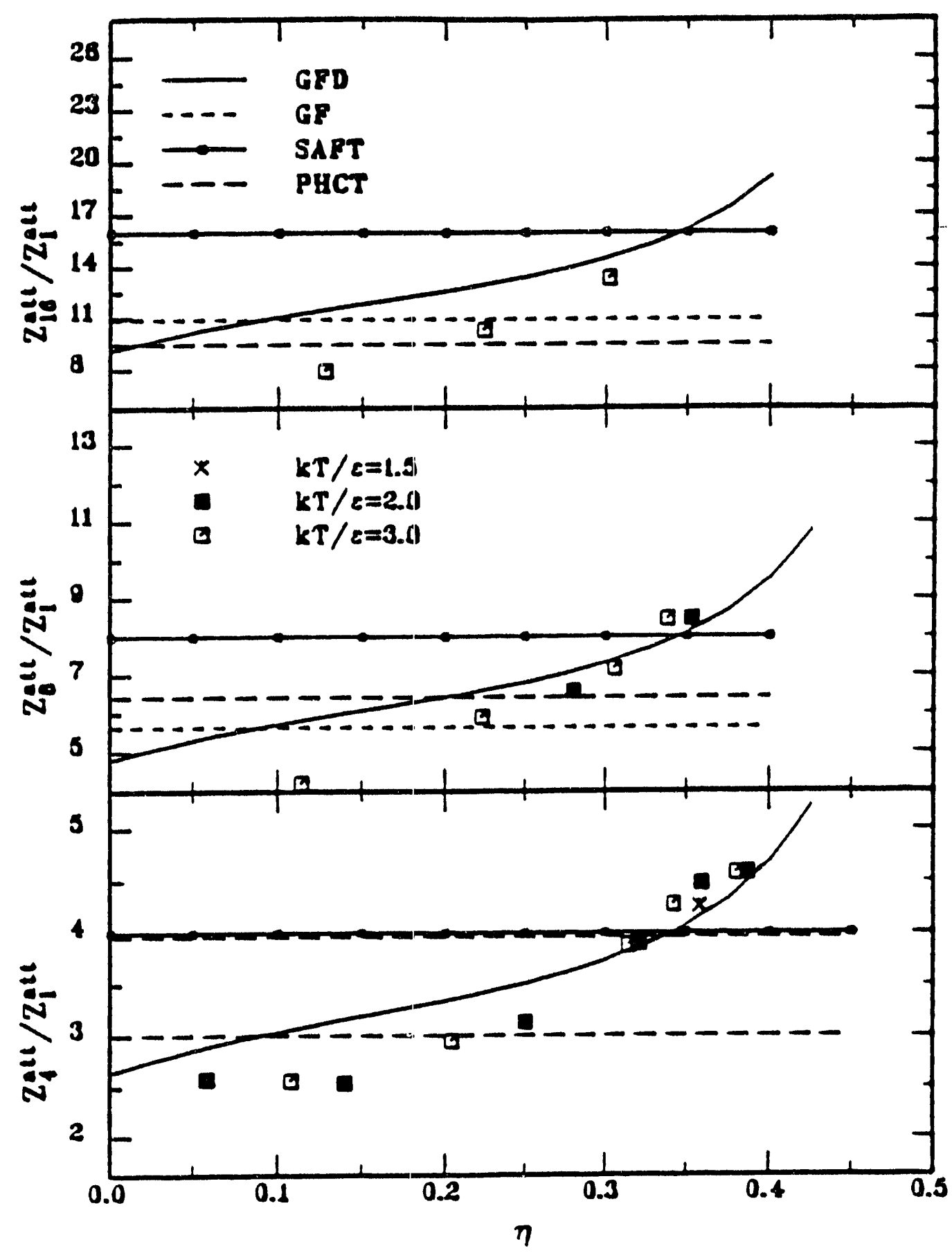

Figure 5. Ratio of attractive contribution to the compressibility factor for chains to that for monomers, calculated by the GF, GFD, SAFT, and PECT models. Simulation data of Yethiraj and Hail (1991b) also are presented. 


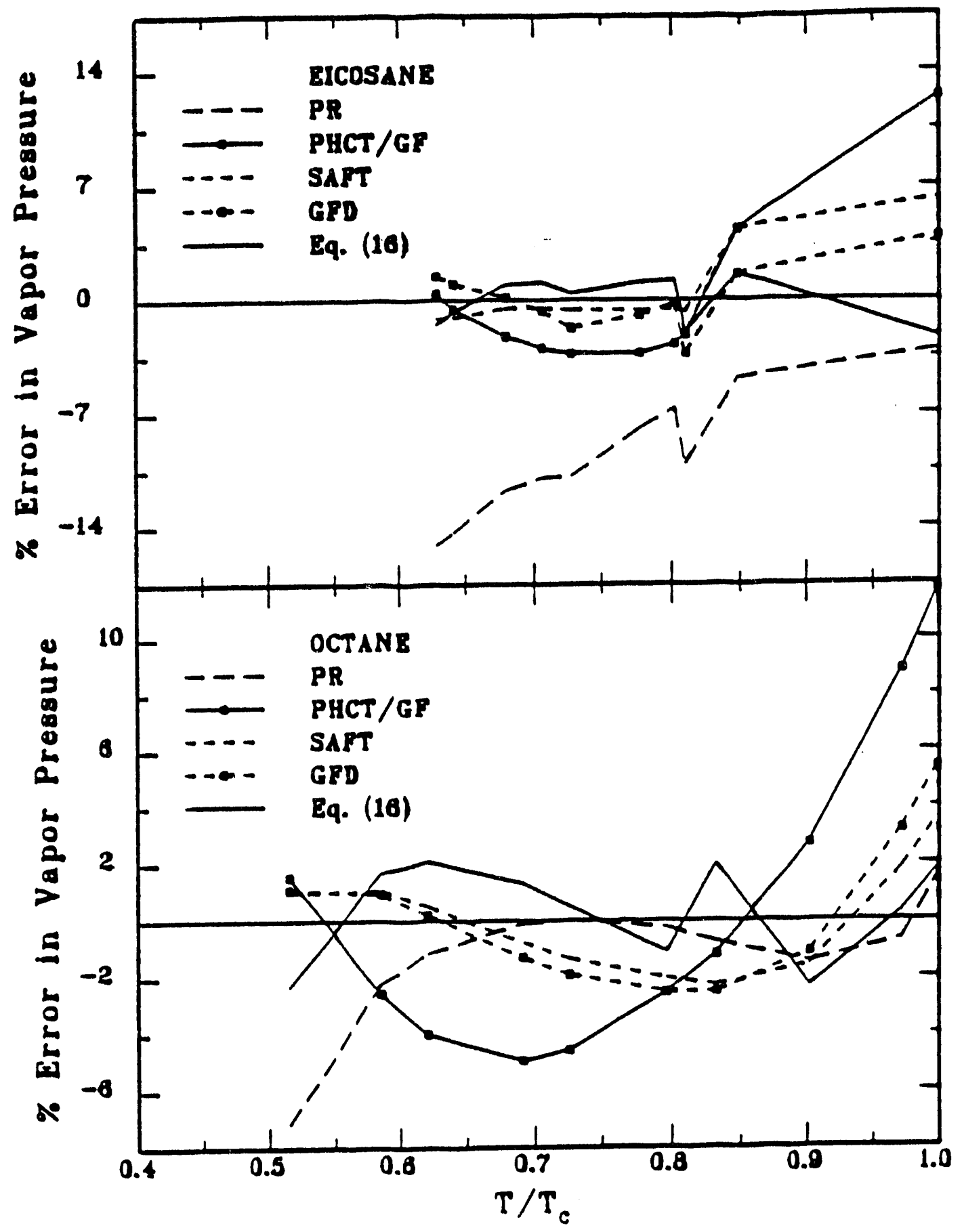

Figure 6. Vapor pressure calculations for octane and eicosane with various equations of state. 
We have shown that the second virial coefficients for spherical molecules, calculated from the square-well potential, are not in good agreement with experimental data for argon. We have developed a new expression for the second virial coefficient for spheres, with a form similar to that from the square-well potential, but more accurate when compared with experimental data. This is very important, since phase equilibrium calculations in mixtures, especially calculations of dew points, are very sensitive to the second virial coefficients.

In order to extend this model to chain molecules, one has to make assumptions about the type of interaction between the molecules at very low densities. At moderate and high densities, chain molecules can be treated using a segment-segment approach. However, this is not the case at low densities. At low densities, interactions occur on a molecule-mo!ecule basis; in this case, the chain molecule can be viewed as a large "equivalent" square-well sphere whose radius is approximately equal to the radius of gyration of the chain molecule. In addition, since the center of a segment on one chain molecule can fall simultaneously into the attractive wells of several segments on a different molecule, the well depth of the large "equivalent" square-well sphere is not simply related to the well depth of a single square-well segment. This behavior is taken into account through the introduction of a new parameter, which is fitted to data for the second virial coefficient of chain molecules. Figure 7 shows the results from fitting our model to second virial coefficient data for dimers, 8-mers and 16-mers, calculated from computer simulations (Wichert and Hall, 1993). One sees that the fits are very good. Very good results also are obtained when we fit experimental second virial coefficient data for normal alkanes. This is shown in Figure 8. We plan to incorporate this expression for the second virial coefficient into our equation of state for chain molecules. This will significantly improve the phase equilibrium calculations in multicomponent systems. 


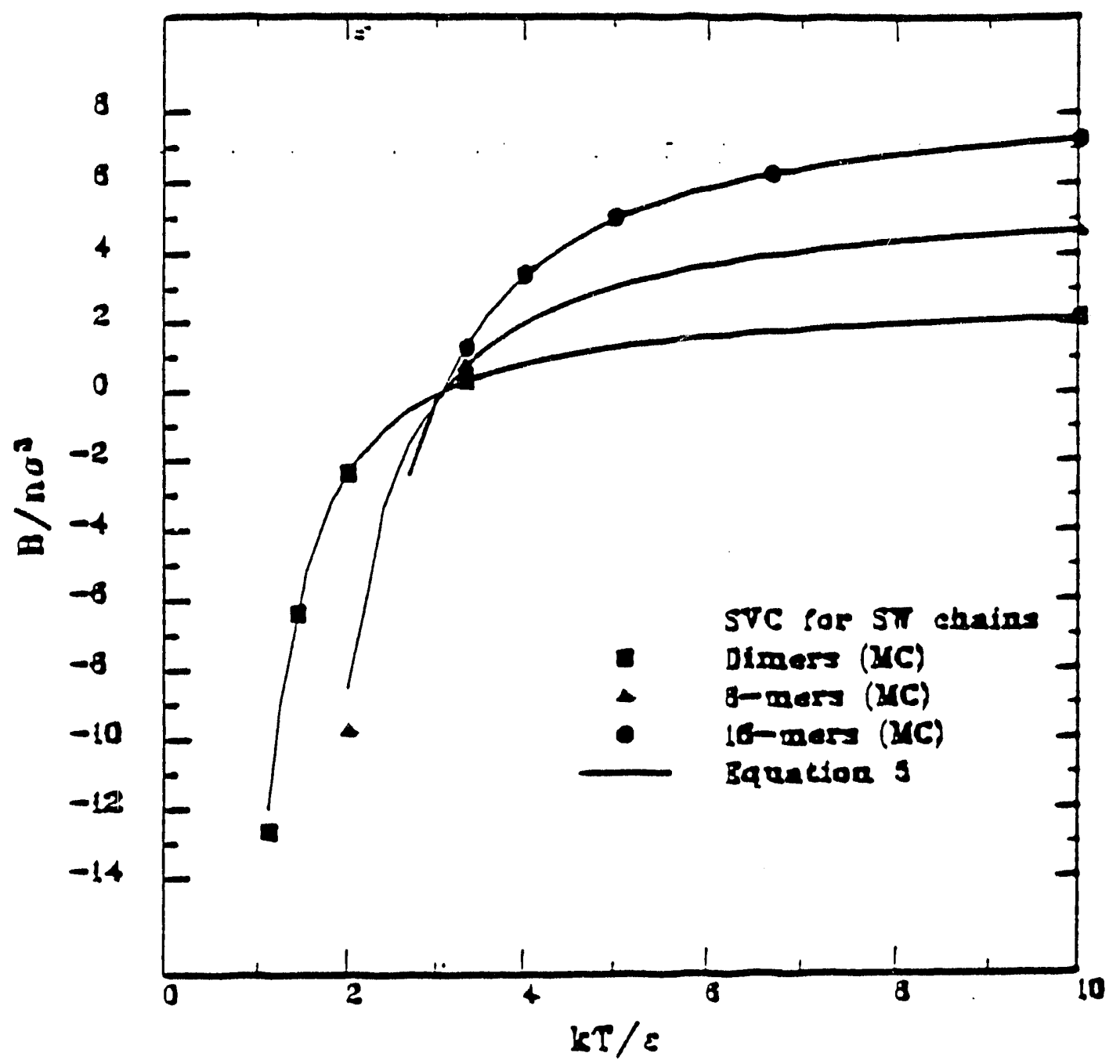

Figure 7. Second virial coefficients for square-well dimers, 8-mers, and 16-mers: computer simulation data (Wichert and Hall, 1993), and correlation with our model. 


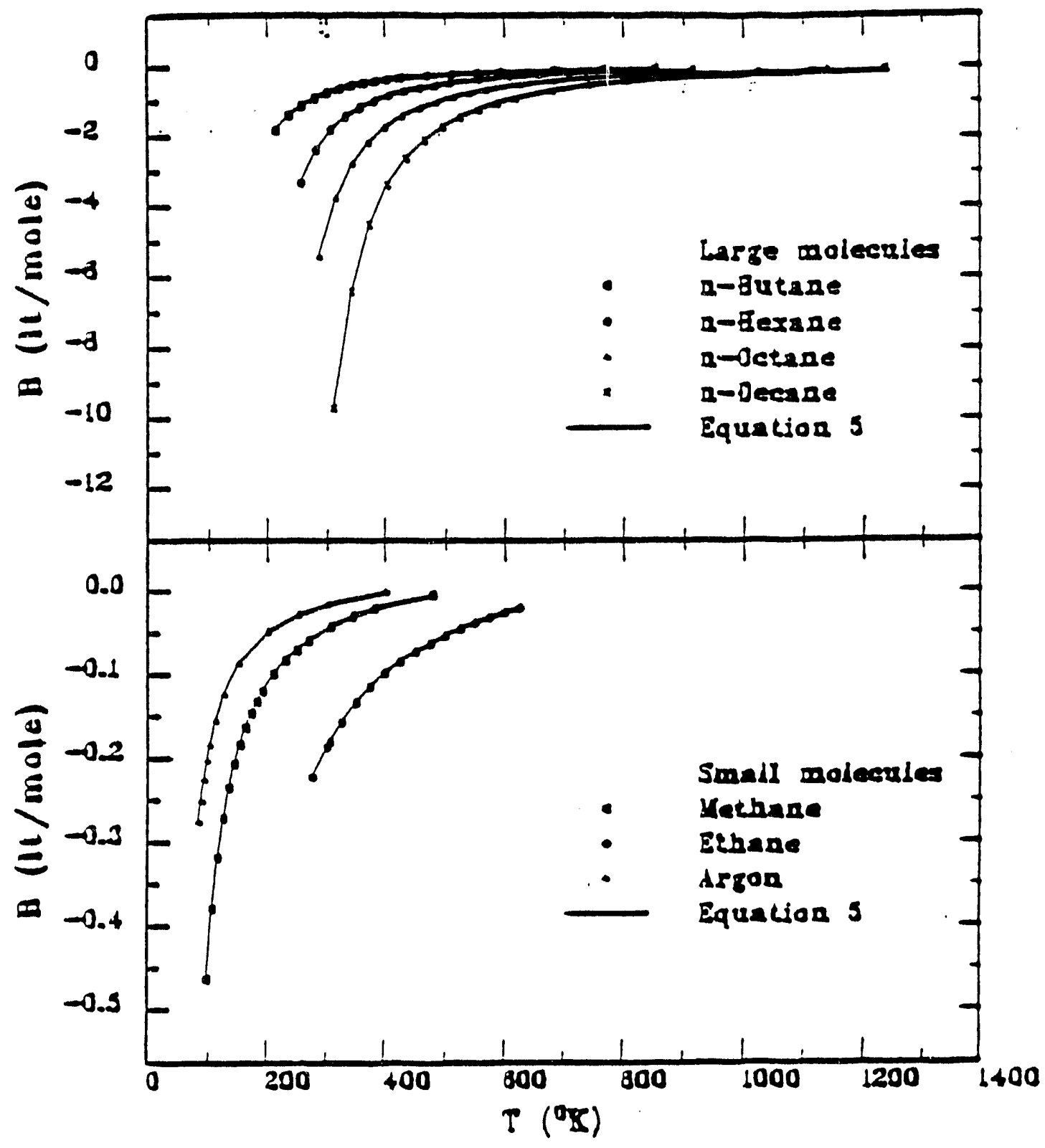

Figure 8. Experimental second virial coefficient data for small and large molecules, and correlation with our model. 


\section{Thermodynamics of Associating Systems}

Over the course of the current contract period, we have extended our model for associating systems to treat water and water-hydrocarbon systems. Modeling the thermodynamic properties and phase equilibria of mixtures of water with aliphatic and aromatic hydrocarbons is a difficult and challenging problem, since such systems show extremely non-ideal behavior that results in limited miscibility over a broad range of conditions. Hydrogen bonding between water molecules results in clusters that can extend over many coordination shells. Each water molecule has four bonding sites, and so each molecule can form one to four hydrogen bonds. The strong hydrogen bonding tendency of water results in the unusual thermodynamic properties of pure water compared with other compounds of similar size. In mixtures, the clustering of water molecules results in limited mutual solubility of water with compounds that do not hydrogen bond.

In previous contract periods, we have developed a rigorous thermodynamic model for associating systems (Ikonomou and Donohue, 1986). This model, (APACT: associated-perturbed-anisotropic-chain theory), is able to give accurate description of the phase equilibria for associating systems, such as alcohols and ketones. However, the results for pure water and water mixtures were not as accurate. This is because APACT accounts for hydrogen bonding through a two-site association model, which is adequate for alcohols and ketones but inadequate for water.

Wertheim (1986a,b), using perturbation theory with a potential function that mimics hydrogen bonding, developed a statistical mechanical model for systems with a repulsive core and multiple hydrogen bonding sites. Economou and Donohue (1991a) showed that Wertheim's perturbation theory and chemical theory (APACT) have essentially identical hydrogen bonding terms. Therefore, we have used this equivalence to derive a three-site APACT model for water. We chose the three-site model instead of a foursite model, since recent experimental results (Wei et al., 1991) show that in clusters of 


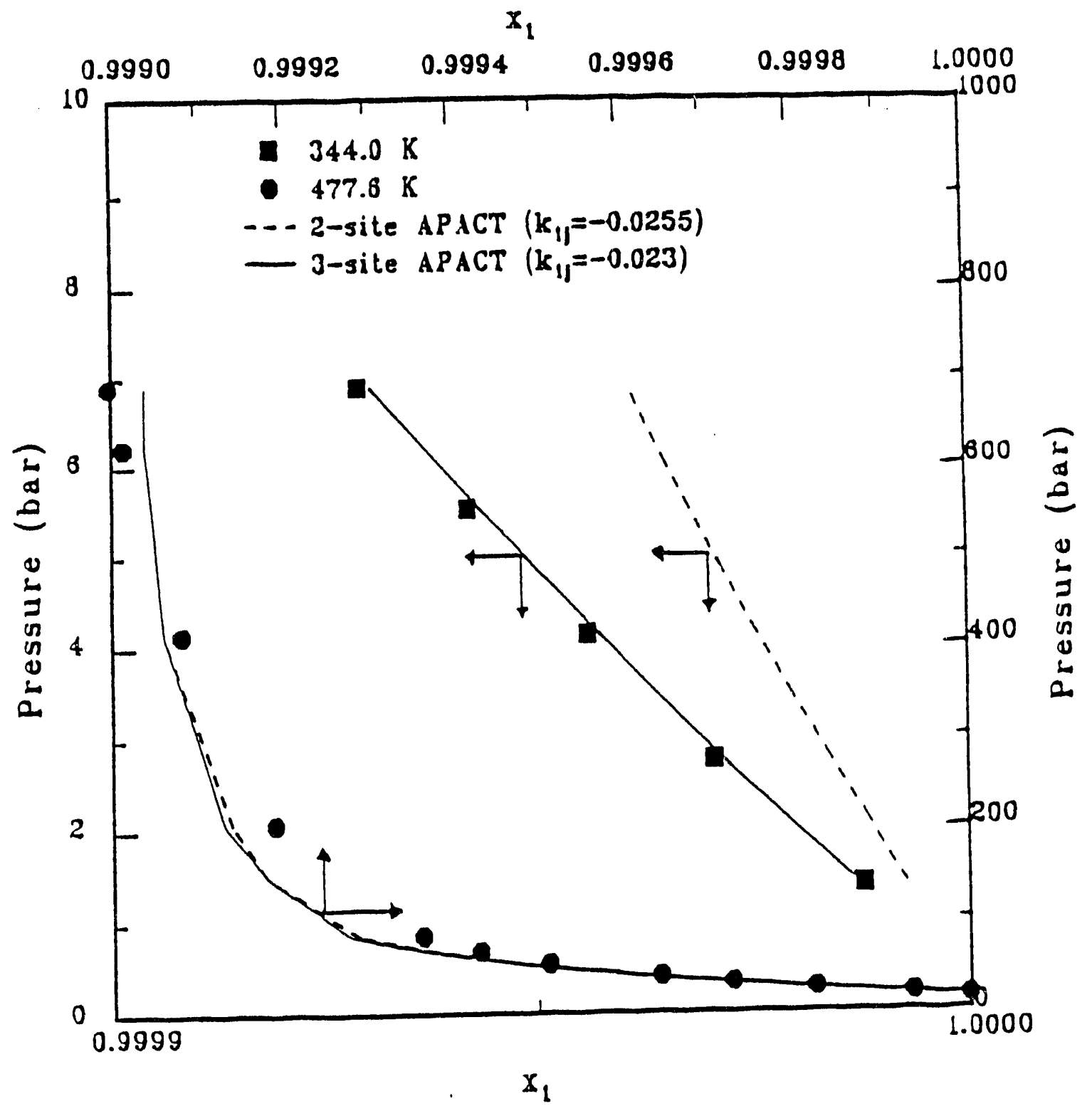

Figure 9. Experimental (points) and calculated (lines) bubble-point values for the VLE of water (1)-butane (2) mixture at 344 and $477.6 \mathrm{~K}$. 
activity is estimated from the concentration of the compound. However, this approximation is not correct at conditions of high pressure and thus leads to erroneous results.

In this work, we developed a method to calculate the activity of a compound over the entire temperature and pressure range using an equation of state. This method has been used widely to calculate thermodynamic properties and phase equilibria of associating fluids (Ikonomou and Donohue, (1986); Elliott et al., (1990)). In this approach, the fugacity coefficient, which is a measure of the non-ideality of the system, is expressed in terms of the equation of state. The advantage of this method is that it calculates accurately the extent of reaction and at the same time it allows prediction of the phase equilibria for the system.

The technique presented is general for multireaction equilibria for any equation of state. Three cubic equations of state were presented. The Redlich-Kwong-Soave (RKS, Soave, 1972) and the Peng-Robinson (PR, Peng and Robinson, 1976) equations of state are the most popular cubic equations of state for thermodynamic and phase equilibria calculations. A cubic equation of state developed recently by Elliott et al., (1990), the Elliott-Suresh-Donohue (ESD) equation of state, was presented also. The ESD was derived based on molecular simulation studies and so it has a semi-theoretical basis. The method was applied to the water-gas shift reaction using the RKS and $P R$ equations of state to calculate the extent of reaction as a function of temperature and pressure. More importantly, the method is able to predict condensation of water for a wide range of temperatures and pressures. In addition, the proposed method was applied to the decomposition of methylcyclohexane which is a strong function of temperature and pressure. Finally, the method was used to calculate the extent of the reaction of carbon monoxide with hydrogen sulfide forming carbonyl sulfide and hydrogen.

Figure 10 illustrates the extent of the water-gas shift reaction as a function of temperature and pressure, calculated with the RKS and PR equations of state. This chemi- 


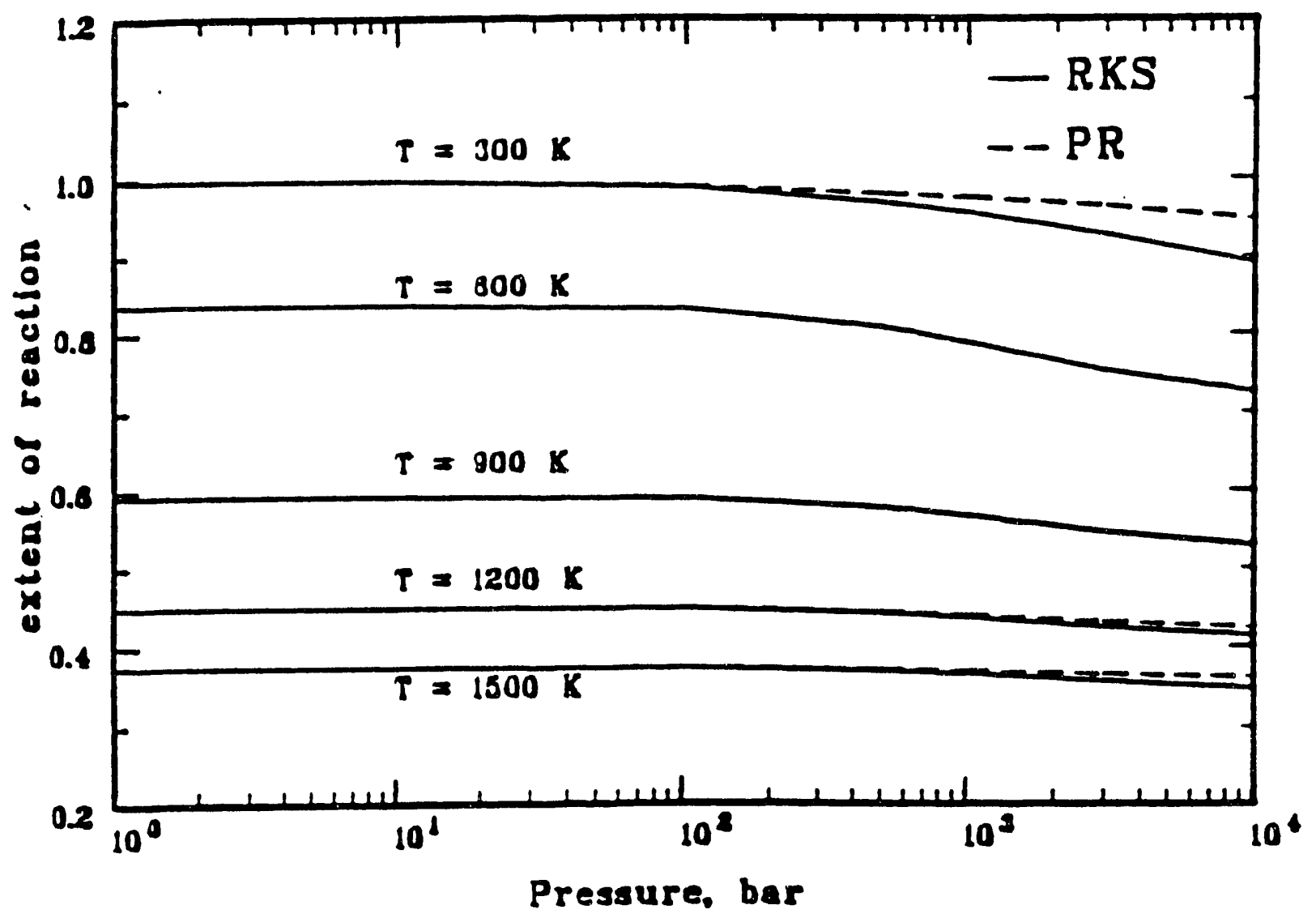

Figure 10. Extent of the water-gas shift reaction from the RKS and the PR equations of state. 
cal reaction is described by the expression:

$$
\mathrm{CO}+\mathrm{H}_{2} \mathrm{O} \rightleftharpoons \mathrm{H}_{2}+\mathrm{CO}_{2}
$$

At low (room) temperatures, the reaction goes almost to completion. Since the reaction is exothermic, at higher temperatures the conversion decreases. In addition, one sees that at low pressures, the reaction is independent of pressure. At pressures higher than 100 bar, the nonidealities of the system have a significant effect on the equilibrium of the mixture. It is interesting to note that at low temperatures, the pressure effect is higher, since at these temperatures, intermolecular forces are strong and highly nonideal.

The water-gas shift reaction takes place in the vapor phase. However at temperatures below the critical temperature of water $\left(T_{c}=647 \mathrm{~K}\right)$, water condensation might occur. In industrial processes, it is very important to know whether the mixture forms one or more phases. A condensed phase might cause significant problems and decrease the efficiency of the process. For the water-gas shift reaction, water condensation would cause problems to compressors, to the catalyst, and to the reactor. Although it is often assumed that the water-gas shift reaction is an ideal-vapor phase reaction ignoring any water condensation, there is a need for a method capable of predicting the phase equilibria of the system simultaneously with the chemical equilibria.

The method proposed in this work is applicable in both the liquid and vapor phases and also can be used to calculate simultaneously chemical and phase equilibria of the system. We developed an iterative algorithm based on phase equilibria fundamentals that takes into account the constraints imposed by the chemical reaction. The equation of state is used to calculate the fugacities of the components in each of the phases. In Figure 11, the region of temperature and pressure where water condenses for the watergas shift reaction is shown. Each of the two lines, corresponding to the predictions from the RKS and PR equations of state, divide the temperature-pressure space into two areas. For temperatures and pressures at the upper left part of the figure, liquid water forms whereas at lower temperatures and pressures all the compounds are in the vapor 
phase. As the temperature increases, the boundary pressure for liquid formation increases and eventually only one vapor phase exists over the entire pressure range. Although the predictions from RKS and PR agree qualitatively, the boundary pressure predicted from the PR has a much higher slope than the boundary pressure predicted from the RKS equation.

\section{EXPERIMENTS}

While most of our work on this research project is theoretical and computational, certain questions can be answered only through laboratory experiments. In addition, experimental results can be used to check the validity and accuracy of the theoretical models that we developed. Below, we present in detail some of the important aspects of our experimental procedures and accomplishments.

\section{Self-Association of Aliphatic Alcohols}

A number of industrially important systems contain molecules, such as alcohols, that hydrogen bond. To correlate the thermodynamic properties of such systems, assumptions must be made in modeling the chemical equilibrium. One approach is to as-

sume an infinite equilibria model, where linear chains are formed having $1,2, \ldots, \mathrm{n}$ associated monomers. Another approach is to assume that monomers, dimers, and tetramers are the dominant species formed. It is useful to determine if these assumptions for the equilibrium models and constants are reasonable. FTIR spectroscopy has been used to study the chemical association of compounds. Analysis of FTIR spectroscopic data enables one to determine the the species present in a mixture, the equilibrium constants, and if the assumptions made about the equilibrium model are valid.

Figure 12 shows the spectrum of a binary mixture composed of 1-nonanol and ndecane for increasing concentration of 1-nonanol. At very low alcohol concentration, 


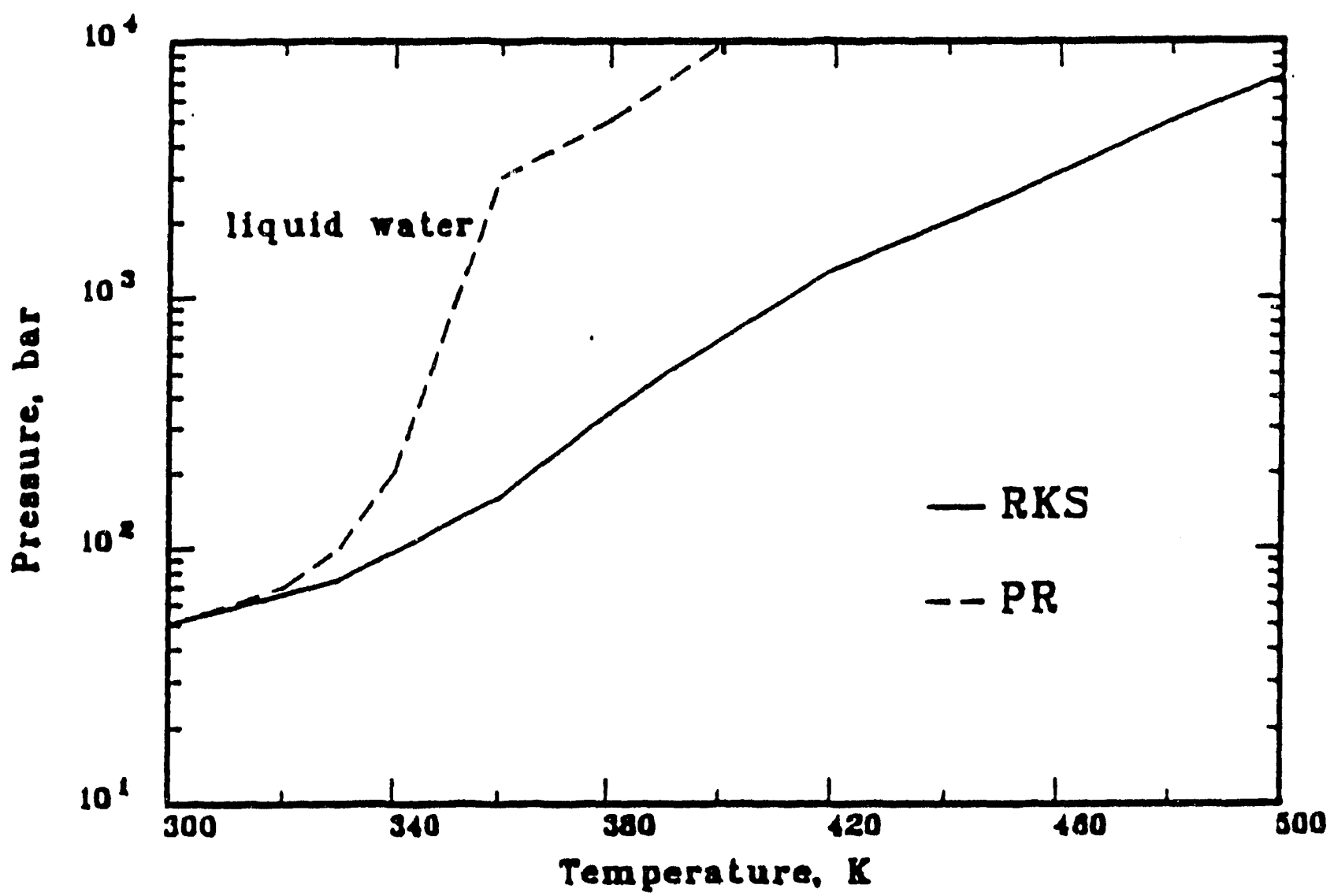

Figure 11. Phase equilibria predictions for the water-gas shift reaction from the RKS and the PR equations of state. At bigh pressures and low temperatures, water condenses. 


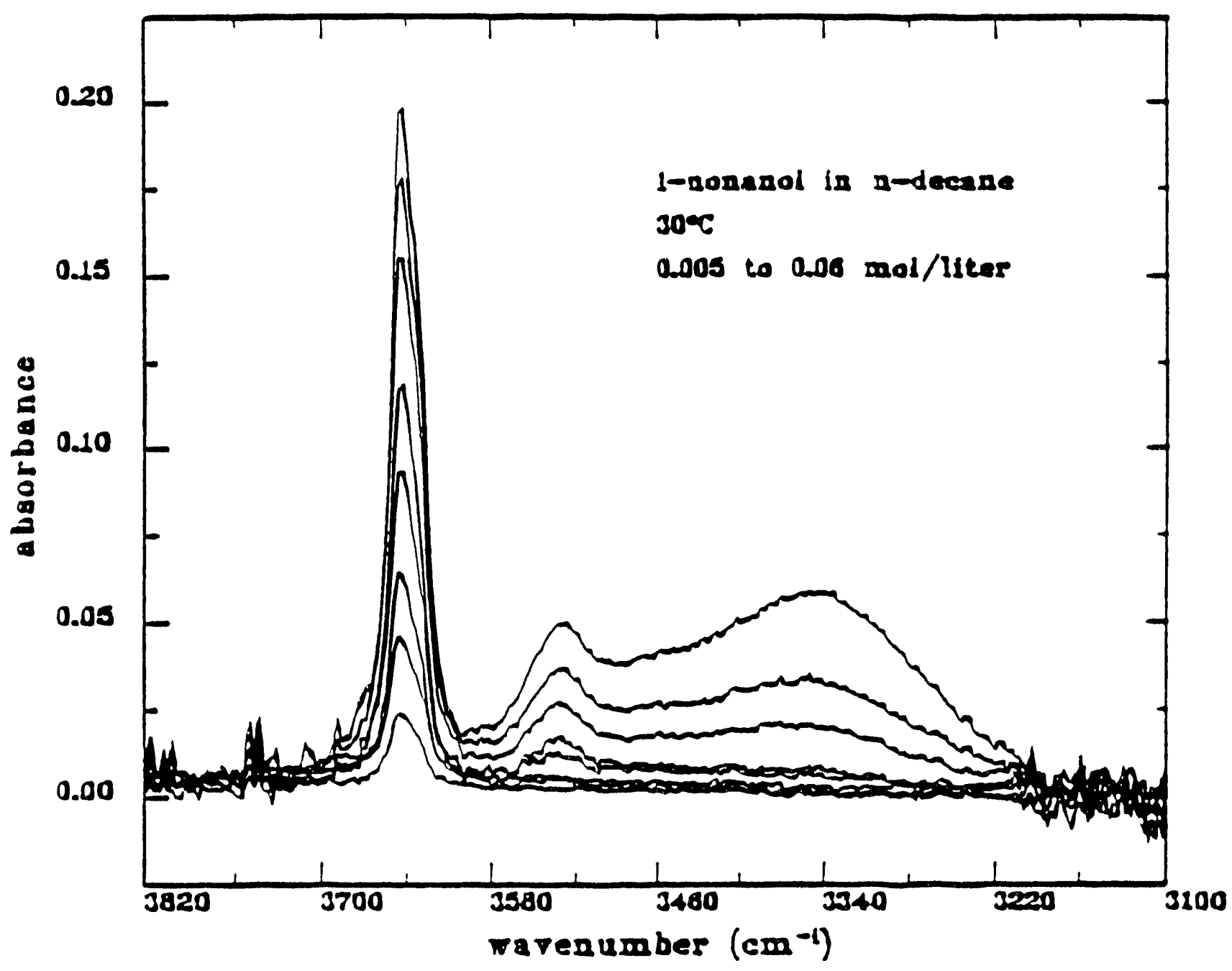

Figure 12. Spectrum of 1-nonanol/n-decane at $30^{\circ} \mathrm{C}$ at different 1 -nonanol concentrations. 
there is only one peak, due to the fundamental stretch of the unbonded $O H$ group. As the alcohol concentration increases, the intensity of the unbonded peak also increases. Second, additional peaks appear and grow in size as the 1-nonanol concentration is increased. These peaks correspond to hydrogen bonds that form between 1-nonanol molecules, and occur at lower wavenumbers than the unbonded band. As Figure 12 shows, these broad peaks are not present at the lower concentrations and are due to the selfassociation of 1-nonanol.

We have applied a novel data analysis technique in order to determine the areas under the different peaks (that often highly overlap). Therefore, the concentrations of the various complexes formed with the hydrogen bonding of 1 -nonanol molecules can be evaluated. This enables us to estimate the equilibrium constants of the "chemical reactions" that result in the formation of the complexes present in the system.

Shown in Figure 13 are the fits of the area versus concentration data for the monomeric peak. The infinite equilibria model was used to determine the equilibrium constant in each case. This involves regressing the enthalpy and entropy of association, since the equilibrium constant is a function of both these quantities. Reasonable fits of the data are obtained within approximately 5 percent difference between the fit and the data points.

\section{Spectroscopy in High-Pressure Fluids}

In addition to spectroscopic measurements of liquids at atmospheric pressure, we also examined the Lewis acid-base interactions and solvent behavior of high-pressure fluids, such as carbon dioxide and ethane. Through the use of well-characterized probes, insight into the nature of high-pressure fluid solvents is found by spectroscopically examining the probes in the high-pressure fluid. This is very useful, since high-pressure and supercritical fluids are widely used in numerous practical applications and therefore, knowledge of their physical and chemical properties is very important. 


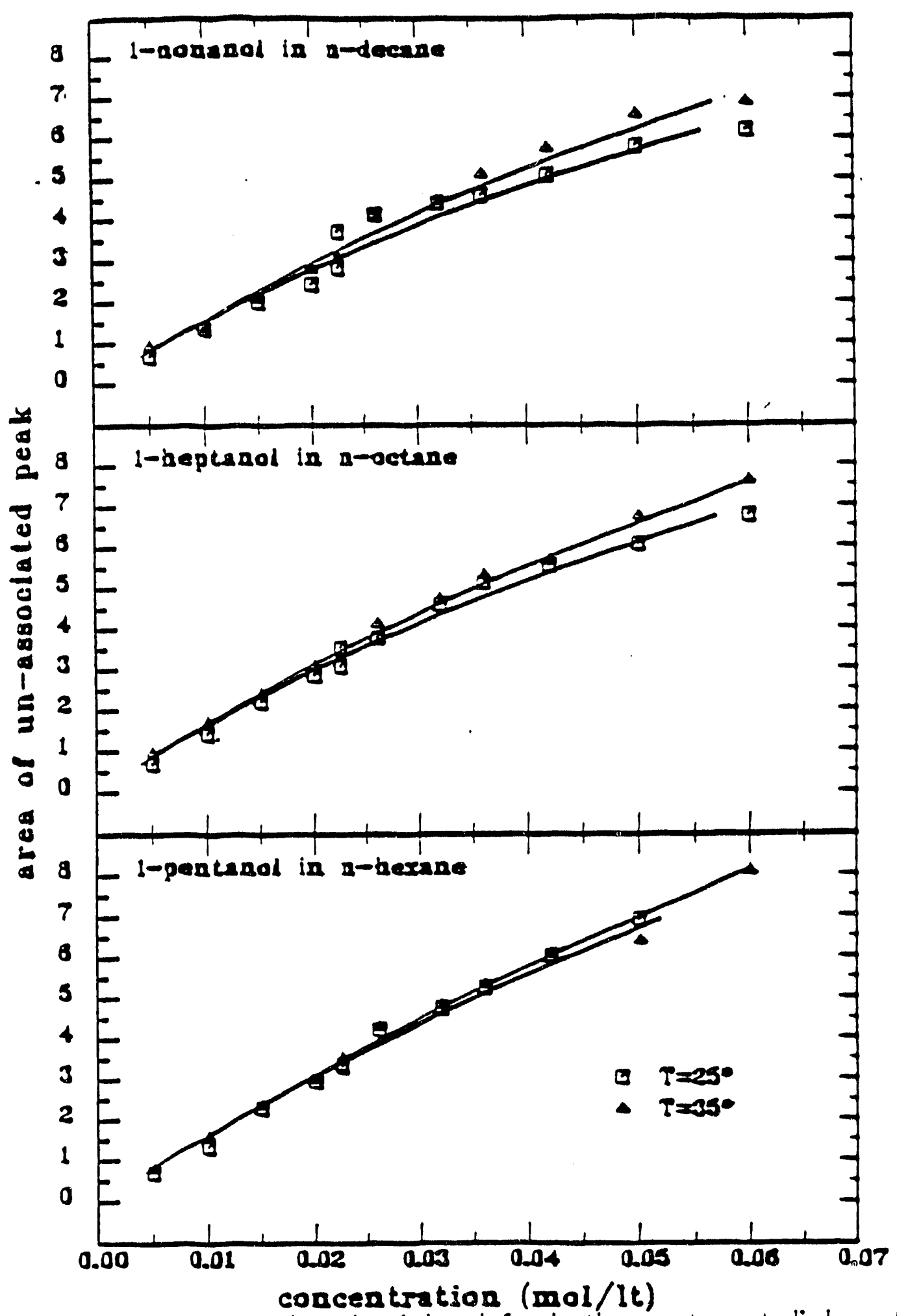

Figure 13. The areas of the unbonded peak for the three systems studied as a function of the concentration and the temperature are shown along with the fits obtained from data regression using the infinite equilibria model. 
Over the course of the current contract period, we have used FTIR spectroscopy to investigate the behavior of ethane and carbon dioxide at high pressures. For this purpose, we have designed and built a high-pressure spectroscopic apparatus. Three probe molecules with known properties have been used (methanol, chloroform, and methanold).

Ethane is not expected to hydrogen bond with any of the probe molecules used in this work. This also was supported by our results. Ethane behaves like inert solvents, such as carbon tetrachloride, and no association between ethane and any of the probes was observed. On the other hand, there has been some controversy about carbon dioxide and its acid-base properties. Figure 14 shows the spectra for the O-D stretch of deuterated methanol in liquid carbon dioxide at room temperature and three different pressures. If a qualitative comparison is made with spectra of methanol-d in inert solvents (such as $\mathrm{CCl}_{4}$ ), we find that the position and shape of the peak is similar to those in high-pressure $\mathrm{CO}_{2}$. Therefore, using a probe that is very sensitive to the solvent environment and can potentially hydrogen bond with carbon dioxide, we find no qualitative evidence that hydrogen bonds are being formed.

Current research on high-pressure fluid spectroscopy is being conducted in order to investigate the enhancement of the solubility of solutes in the high-pressure fluid, when a third component is inserted into the system as an entrainer cosolvent. In particular, we are examining the increase of the solubility of methanol-d in carbon dioxide at high pressures, when acetone (which can hydrogen bond with methanol- $d$ ) is added as a cosolvent. In the future, we plan to add temperature control in the high-pressure apparatus, so that the supercritical region is obtained.

\section{Spectroscopy in Amphiphilic Fluids}

In order to test the applicability of our model for amphiphiles, we are currently conducting laboratory experiments using FTIR spectroscopy. During the first phase of 


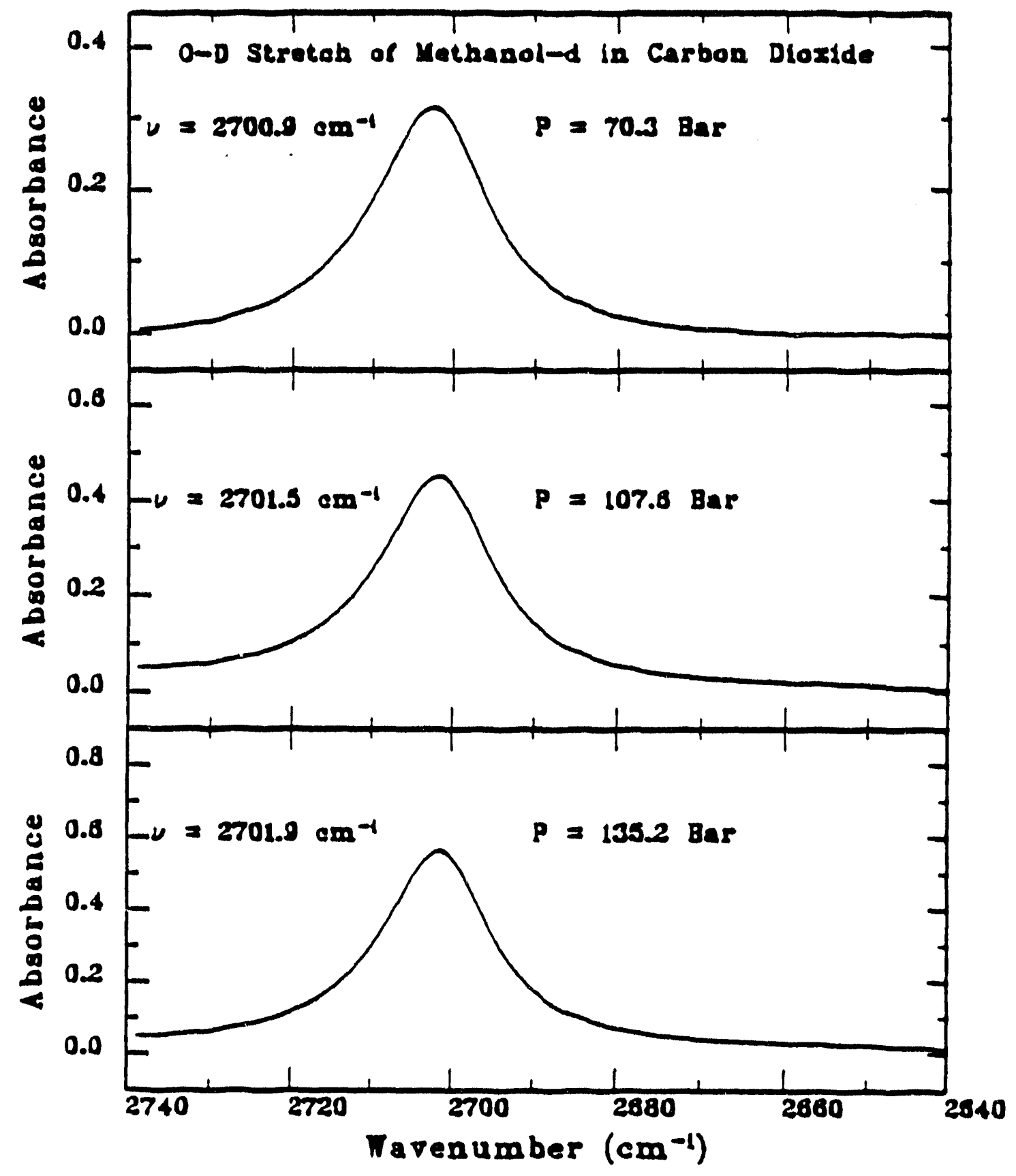

Figure 14. Absorbance versus wavenumber for the O-D stretch of methanol-d in highpressure carbon dioxide at room temperature and three different pressures. 
this project, we are synthesizing amphiphiles with fluorocarbon and hydrocarbon ends (i.e., $\left.C F_{3}\left(\mathrm{CF}_{2}\right)_{a}\left(\mathrm{CH}_{2}\right)_{b} \mathrm{CH}_{3}\right)$. This type of amphiphile offers two advantages. First, it interacts only through simple dispersion forces; no hydrogen bonding or site-specific interactions occur. Second, binary fluorocarbon-hydrocarbon mixtures can be easily prepared so that they demix near room temperature. The thermodynamic transition between locally-ordered microphases and a random bulk phase in such hydrofluorocarbon amphiphiles is strongly correlated with the demixing of a similar alkane-perfluoroalkane mixture (Kuespert and Donohue, 1993a, 1993b).

After the synthesis of amphiphilic compounds is finished, we are planning to perform FTIR spectroscopic measurements in order to characterize their behavior, both neat and in solution with hydrocarbons and fluorocarbons. We have performed preliminary experiments using a pair of commercially-available hydrofluorocarbon amphiphiles (perfluorobutyl- and perfluorohexyl-ethene) and identified several infrared spectral features that show apparent solvatoochromic shifts. We intend to perform a series of experiments that will enable us to determine microphase compositions and polarities and to study transitions from ordered to disordered states more effectively.

\section{Literature Cited}

Beret, S. and J. M. Prausnitz, "Perturbed-Hard-Chain Theory: An Equation of State for Fluids Containing Small or Large Molecules," AIChE J., 21, 1123 (1975).

Chapman, W. G.; Gubbins, K. E.; Jackson, G.; Radosz, M., "New Reference Equation of State for Associating Liquids," Ind. Eng. Chem. Res., 65, 1709 (1990)

Dickman, R. and C. K. Hall, "Equation of State for Chain Molecules: Continuous-Space Analog of Flory Theory," J. Chem. Phys., 85, 4108 (1986).

Dickman, R.; Hall, C. K., "High density Monte Carlo simulations of chain molecules: Bulk equation of state and density profiles near walls," J. Chem. Phys., 89 (1988).

Donohue, M. D.; Prausnitz, J. M., "Perturbed Hard Chain Theory for Fluid Mixtures: Thermodynamic Properties for Mixtures in Natural Gas and Petroleum Technology," AIChE J., 24, 849 (1978). 
Douglas, J. F.; Roovers, J.; Freed, K. F., "Characterization of Branching Architecture through "Universal" Ratios of Polymer Solution Properties", Macromolecules, 23, 4168 (1990).

Dudowicz, J.; Freed, K. F.; Madden, W. G., "Role of Molecular Structure on the Thermodynamic Properties of Melts, Blends, and Concentrated Polymer Solutions. Comparison of Monte Carlo Simulations with the Cluster Theory for the Lattice Model", Macromolecules, 23, 4803 (1990).

Economou, I. G. and M. D. Donohue, "Chemical, Quasi-Chemical and Perturbation Theories for Associating Fluids," AIChE J., 37, 1875 (1991a).

Elliott, J. R., S. J. Suresh, and M. D. Donohue, "A Simple Equation of State for NonSpherical and Associating Molecules," Ind. Eng. Chem. Res., 29, 1476 (1990).

Freed, K. F.; Bawendi, M. G., "Lattice Theories of Polymeric Fluids", J. Phys. Chem., 93, 2194 (1989).

Freed, K. F.; Pesci, A. I., "Computation of the Cross-Link Lependence of the Effective Flory Interaction Parameter $\chi$ for Polymer Networks", Macromolecules, 22, 4048 (1989).

Gao, J.; Weiner, J. H., "Contribution of covalent bond force to pressure in polymer melts," J. Chem. Phys., 01, 3168 (1989).

Honnell, K. G.; Hall, C. K., "A new equation of state for athermal chains," J. Chem. Phys., 90, 1841 (1989).

Ikonomou, G. and M. D. Donohue, "Thermodynamics of Hedrogen Bonded Molecules: The Associated-Perturbed-Anisotropic-Chain Theory," AIChE J., 32, 1716 (1986).

Joslin, C. G.; Gray, C. G.; Chapman, W. G.; Gubbins, K. E., "Theory and Simulation of Associating Liquid Mixtures. II.", Molec. Phys., 62, 843 (1987).

Kuespert, D. R. and M. D. Donohue, "Local Order and Microphase Formation in Fluids Containing Asymmetric Molecules," Langmuir, 8, 431 (1993a).

Kuespert, D. R. and M. D. Donohue, "Local Ordering in Asymmetric Chain Fluids," J. Chem. Phys., 98, 9782 (1993b).

Panayiotou, C.; Sanchez, I. C., "Hydrogen Bonding in Fluids: An Equation of State Approach", Submitted to J. Phys. Chem. (1991).

Peng, D. Y. and D. B. Robinson, "A New Two Constant Equation of State," Ind. Eng. Chem. Fundam., 15, 59 (1976). 
Soave, G., "Equilibrium Constants from a Modified Redlich-Kwong Equation of State," Chem. Eng. Sci., 27, 1197 (1972).

Wei, S., Z. Shi, and A. W. Castleman, Jr., "Mixed Cluster Ions as a Structure Probe: Experimental Evidence for Clathrate Struucture of $\left(\mathrm{H}_{2} \mathrm{O}\right)_{20} \mathrm{H}^{+}$and $\left(\mathrm{H}_{2} \mathrm{O}\right)_{21} \mathrm{H}^{+}, " \mathrm{~J}$. Chem. Phys., 94, 3268 (1991).

Wertheim, M. S., "Fluids with Highly Directional Attractive Forces. II. Thermodynamic Perturbation Theory and Integral Equations", J. Stat. Phys., 35, 35 (1984).

Wertheim, M. S., "Fluids with Highly Directional Attractive Forces. III. Multiple Attraction Sites", J. Stat. Phys., 42, 459 (1986a).

Wertheim, M. S., "Fluids with Highly Directional Attractive Forces. IV. Equilibrium Polymerization", J. Stat. Phys., 42, 477 (1986b).

Wertheim, M. S., "Fluids of Dimerizing Hard Spheres, and Fluid Mixtures of Hard Spheres and Dispheres", J. Chem. Phys., 85, 2929 (1986c).

Wertheim, M. S., "Thermodynamic Perturbation Theory of Polymerization", J. Chem. Phys., 87, 7323 (1987).

Wichert, J. M. and C. K. Hall, "Computer Simulations of Second Virial Coefficients for Square-Well Chains," Macromolecules, submitted for publication (1993).

Yethiraj, A.; Hall, C. K., "Generalized Flory equations of state for square-well chains," J. Chem. Phys., 95, 8494 (1991a).

Yethiraj, A. and C. K. Hall, "Square-Well Chains: Bulk Equation of State Using Perturbation Theory and Monte Carlo Simulations of the Bulk Pressure and of the Density Profiles Near Walls," J. Chem. Phys., 95, 1999 (1991b). 


\section{Publications Resulting from this Research Program During the Current Contract Period}

Walsh, J. M., G. Jin, and M. D. Donohue, "Thermodynamics of Short Chain Polar Compounds," Fluid Phase Equil., 65, 209 (1991).

Economou, I. G., Y. Cui, and M. D. Donohue, "Hydrogen Bonding in Polymer - Solvent Mixtures," Macromolecules, 24(18), 5058 (1991a).

Economou, I. G., P. Vimalchand, and M. D. Donohue, "Measurement of Infinite Dilution Activity Coefficients Using High Performance Liquid Chromatography," Fluid Phase Equil., 68, 131 (1991b).

Economou, I. G. and M. D. Donohue, "Chemical, Quasi-Chemical and Perturbation Theories for Associating Fluids," AIChE J., 37, 1875 (1991a).

Economou, I. G. and M. D. Donohue, "On Chemical Theory Expressions for Mixtures of Amphoteric Components," AIChE J., 37, 795 (1991b).

Vimalchand, P., A. Thomas, I. G. Economou, and M. D. Donohue, "Effect of HardSphere Structure on Pure-Component Equation of State Calculations," Fluid Phase Equil., 73, 39 (1992).

Reilly, J. T., C. Kim, A. B. Clark, and M. D. Donohue, "High Pressure Vapor-Liquid Equilibria of Aromatic Hydrocarbons with Carbon Dioxide and Ethane," Fluid Phase Equil., 73, 81 (1992a).

Economou, I. G. and M. D. Donohue, "Thermodynamic Inconsistencies in and Accuracy of Chemical Equations of State for Associating Fluids," Ind. Eng. Chem. Res., 31, 1203 (1992a).

Campbell, S. W., I. G. Economou, and M. D. Donohue, "Closed-Form Expressions for 'Chemical Theory' of Associating Mixtures," AIChE J., 38, 611 (1992).

Bokis, C. P. and M. D. Donohue, "Shape Parameters and the Density Dependence of Hard-Chain Equations of State," AIChE J., 38, 788 (1992).

Reilly, J. T., J. M. Walsh, M. L. Greenfield, and M. D. Donohue, "Analysis of FT-IR Spectroscopic Data: the Voigt Profile," Spectrochimica Acta, 48A, 1459 (1992b).

Economou, I. G. and M. D. Donohue, "Equation of State with Multiple Associating Sites for Water and Water - Hydrocarbon Mixtures," Ind. Eng. Chem. Res., 31, 2388 (1992b). 
Cui Y. and M. D. Donohue, "Entropy and Energy of Mixing in Polymer Solutions: Simple Expressions that Approximate Lattice Cluster Theory," Macromolecules, 25, 6489 (1992).

Bokis, C. P., M. D. Donohue, and C. K. Hall, "Local Composition Model for SquareWell Chains Using the Generalized Flory Dimer Theory," J. Phys. Chem., 96, 11004 (1992b).

Anderko, A., I. G. Economou, and M. D. Donohue, "Comments on "Thermodynamic Inconsistencies in and Accuracy of Chemical Equations of State for Associating Fluids'," Ind. Eng. Chem. Res., 32, 245 (1993).

Thomas, A., J. M. Walsh, and M. D. Donohue, "A Parameterization of the Configurational Energy for Short-Chain Molecules," AIChE J., 39, 193 (1993a).

Bokis, C. P., Y. Cui, and M. D. Donohue, "Thermodynamic Properties of Hard-Chain Molecules," J. Chem. Phys., 88, 5023 (1993a).

Kuespert, D. R. and M. D. Donohue, "Local Order and Microphase Formation in Fluids Containing Asymmetric Molecules," Langmuir, 9, 431 (1993a).

Economou, I. G., M. D. Donohue, and L. W. Hunter, "Equation-of-State Calculations of Chemical Reaction Equilibrium in Non-Ideal Systems," Int. J. Thermophys., 14, 199 (1993).

Kuespert, D. R. and M. D. Donohue, "Local Ordering in Asymmetric Chain Fluids," J. Chem. Phys., 98, 9782 (1993b).

Thomas, A. and M. D. Donohue, "An Equation of State for Chain Molecules Using a Site-Interaction Model: Theory, Simulation and Correlation of Experimental Data," Ind. Eng. Chem. Res., in press (1993b).

Thomas, A. and M. D. Donohue, "Square-Well Dimers of Different Bond Lengths: Theory and Simulation for the Equation of State," Molec. Phys., submitted for publication (1993a).

Bokis, C. P., M. D. Donohue, and C. K. Hall, "Second Virial Coefficients for Chain Molecules," Ind. Eng. Chem. Res., submitted for publication (1993b).

Bokis, C. P., M. D. Donohue, and C. K. Hall, "Application of a Modified Generalized Flory Dimer Theory to Normal Alkanes," Ind. Eng. Chem. Res., submitted for publication (1993c). 
Thomas, A., J. T. Reilly, A. Reyes, C. Y. Chong, and M. D. Donohue, "Analysis of the Self-Association of Aliphatic Alcohols Using Fourier Transform Infrared (FT-IR) Spectroscopy," J. Am. Chem. Soc.; this paper will be submitted before the end of the current contract period (1993b). 

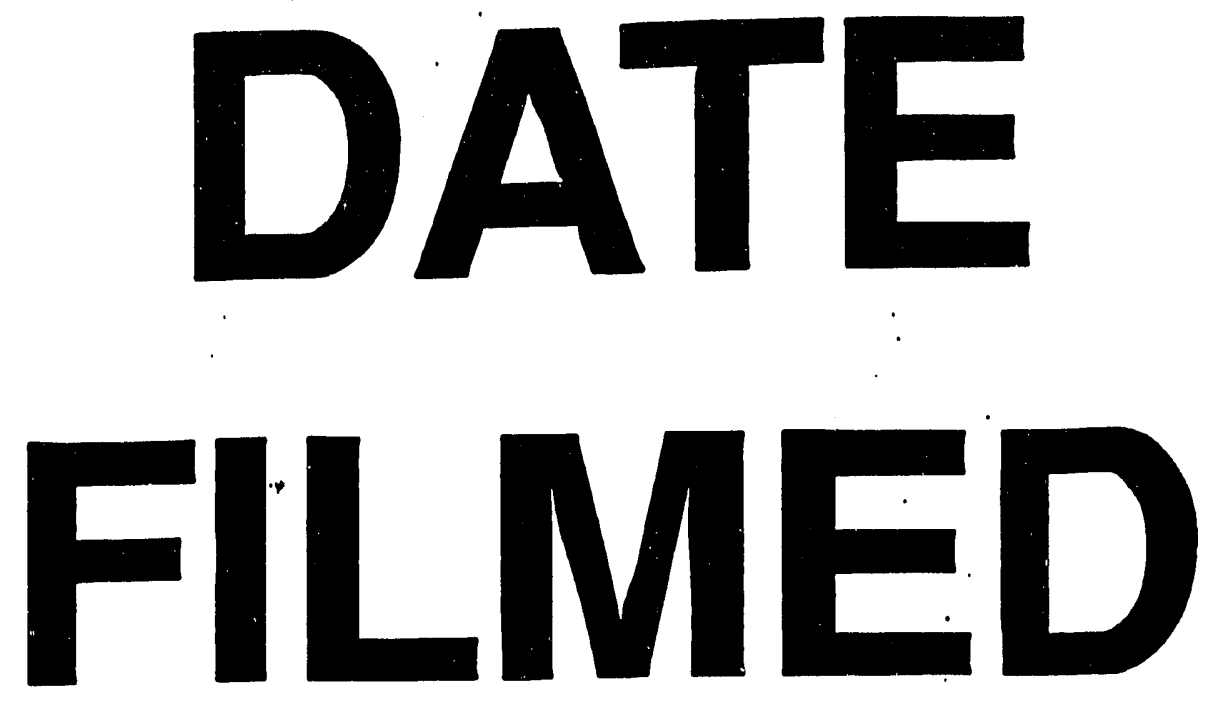

$12 / 10 / 93$
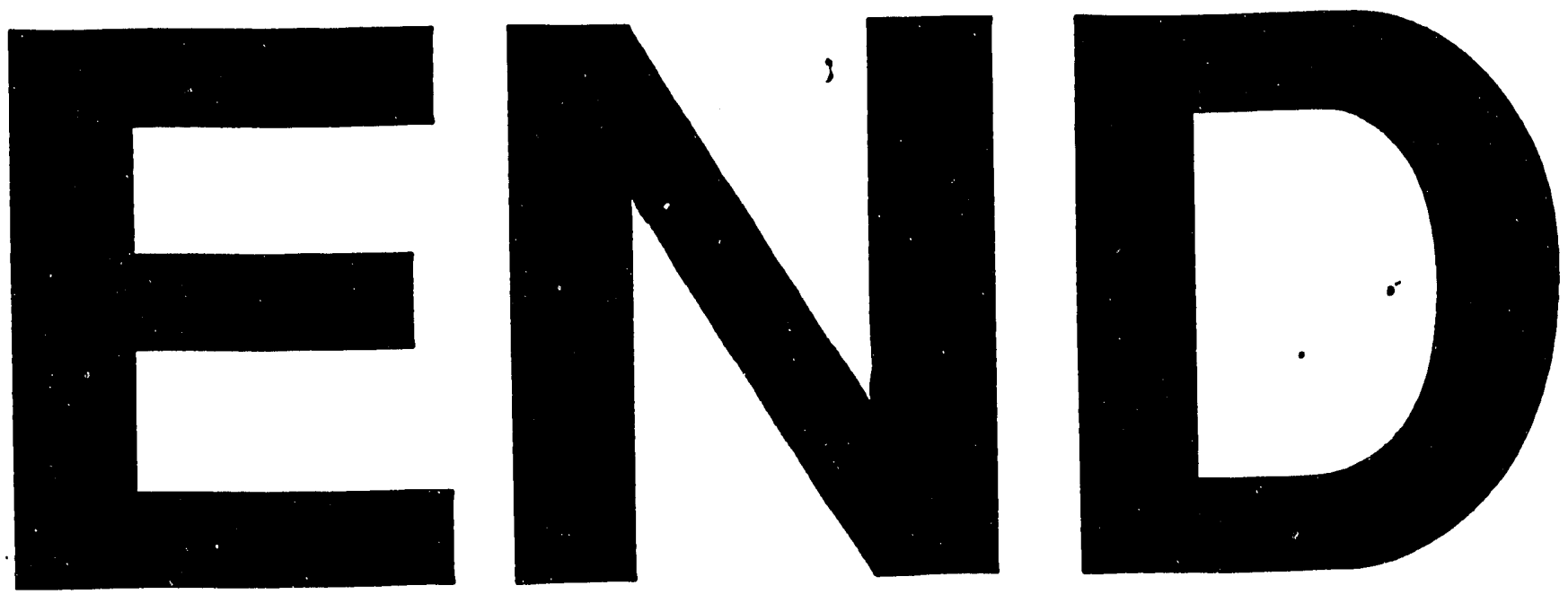
